\title{
Small Angle Neutron Scattering Investigation of Compressed Bentonite Plugs
}

\author{
Tomasz Blach, Heinz-Gerd Holl \\ Center for Coal Seam Gas, University of Queensland, Brisbane, Australia \\ Email: h.holl@uq.edu.au
}

How to cite this paper: Blach, T. and Holl, H.-G. (2019) Small Angle Neutron Scattering Investigation of Compressed Bentonite Plugs. Journal of Minerals and Materials Characterization and Engineering, 7, 230-260.

https://doi.org/10.4236/jmmce.2019.75018

Received: June 26, 2019

Accepted: September 9, 2019

Published: September 12, 2019

Copyright $\odot 2019$ by author(s) and Scientific Research Publishing Inc. This work is licensed under the Creative Commons Attribution International License (CC BY 4.0).

http://creativecommons.org/licenses/by/4.0/ c) (i) Open Access

\begin{abstract}
Bentonite plugs manufactured with and without a polymer binder were studied using Small Angle Neutron Scattering (SANS) and Ultra Small Angle Neutron Scattering (USANS) techniques. We have subjected the samples to hydration by evaporating water and then applied $\mathrm{CD}_{4}$ methane at a Zero Average Contrast (ZAC) pressure (about $48.3 \mathrm{MPa} / 7000 \mathrm{psi}$ ) to measure the accessible and inaccessible pores and channels. The data show that introduction of moisture has an immediate effect on the smallest pores, with swelling preventing access by the methane; however the larger network appears to be little affected by the addition of the moisture. The addition of a binder to the plug creates a greater number of open pores in the micron and above size regime, indicating that the polymer is vital in creating larger channels, which aids the movement of moisture throughout the plug, at the expense of sealing the smallest ones. This suggests that the polymer binder helps in the self-healing process in the clays and makes the structure more stable. The process of plug manufacturing and drying produces a chaotic system of aggregates in the sample, which forms packed clusters $130 \mathrm{~nm}$ in size. Addition of moisture destroys this structure. Addition of water to the dried plug material showed that at 18 weight $\%$ a dual layer of water has formed in the montmorillonite layer of the clay, separating the montmorillonite layers by $17 \AA$ A. Increase to 33 weight\% of water has added an extra layer with basal spacing of $19 \AA$. The clay hydration with vapor and directly deposited liquid appear to delaminate some of the clay affecting the fractality index at the micron sizes. Mechanical compression had a significant effect on the polymerised sample, encouraging larger water-conducting channels to form.
\end{abstract}

\section{Keywords}

SANS, USANS, Bentonite, Porosity, Swelling Process 


\section{Introduction}

Over the last century, a large number of bore wells have been drilled worldwide so that prospecting, utilization and geological assessment of available deposits can be made. As the human population grows, there is an increased pressure to look for new deposits of fresh water, natural gas and oil. The result is increased bore drilling activity worldwide.

A large proportion of these wells become abandoned with time and often leak pollutants into the underground aquifers and the atmosphere, making them a hazard and a source of pollution [1]. Unplugged and badly plugged wells have been identified as major problems facing the resource extraction industry and, as a consequence, almost all state and federal governments around the world require wells to be properly sealed (e.g. [2]). Traditionally, plugs made out of cement have been used for this task. However due to changing local geological conditions (such as those due to fluid and gas movement, seismic activities and varying $\mathrm{pH}$ levels), these plugs can fail, resulting in pollutants reaching underground aquifers and eventually venting into the atmosphere. Therefore more reliable plug technology is needed. Bentonite is a mineral mixture containing primarily the clay mineral montmorillonite that has been used for shallow water well abandonment in a variety of forms [3] and has been slowly gaining acceptance for plugging oil and gas wells [4]. Plugging wells with bentonite is supposed to be cheaper than using concrete plugs [4]. Plugs made of this material are also claimed to be more reliable than those made of concrete. While concrete can fail to seal or later crack due to ground movement or tectonic activity, hydrated bentonite is deformable and hence self-sealing in such conditions.

However, one of the difficulties with using this material is getting bentonite to the bottom of the hole before it sets up. Simply pouring bentonite granules into the well tends to result in early bentonite swelling and bridging soon after it hits the water column. A solution to this problem is to compress the plugging material into bullet-shaped bars using a suitable binder [5]. This retards the hydration kinetics and allows bentonite to be placed at the correct plug location before the swelling occurs. Recent field tests on such plugs showed a lot of promise [6].

Small Angle Neutron Scattering (SANS) and Ultra Small Angle Neutron Scattering (USANS) were chosen as the investigative techniques for this study, as it offers a unique window into the world of the nano and micro structure. Comparative measurements can be done while the sample is exposed to dynamic agents such as moisture, gas and mechanical strain, while the method of probing does not affect these processes. By varying the contrast between various agents, it is also possible to identify structures undergoing change, thus revealing information on the nature of the material under investigation.

\section{Methods}

\subsection{Sample Preparation}

Two, physically identical, but compositionally different plugs, with an outer di- 
ameter of $139.7 \mathrm{~mm}$ were made at the bentonite plug testing facility, University of Queensland, Brisbane, Australia. One plug was compacted using the raw bentonite material, the second one was made out of a bentonite-polymer blend [7]. The mineralogy of the bentonite material is published by Holl \& Scheuermann [8]. An $18 \mathrm{~mm}$ internal diameter plug drill was used to cut a number of cores out of each plug. These cores were then cut into $5 \mathrm{~mm}$ thick disks and placed inside a Teflon former, gently compressed and sanded down to a thickness of about $1 \mathrm{~mm}$. The disks were then placed in aluminum sample holders for vacuum drying.

The raw bentonite sample batches (SA) and the polymer blend (SP) were locked in a vacuum oven set to $70^{\circ} \mathrm{C}$ for a minimum of $24 \mathrm{hrs}$.

\subsection{Hydration Process}

Following the drying of the samples, a controlled amount of water vapor was introduced to each of the discs individually. In order to track where the water ends up in the sample, we carefully prepared a mixture of heavy and distilled water mixture, so that the Scattering Length Density $(S L D)$ matched this liquid with the bentonite. The mixture of $73 \% \mathrm{H}_{2} \mathrm{O}$ and $27 \% \mathrm{D}_{2} \mathrm{O}$ was derived with the help of the NIST SLD calculator software [9] and was then used for all hydrogenation work in this study. The discs were placed in aluminum sample holders and placed in a glass jar on a metal platform to keep the sample above the added liquid water. The amount of water needed to achieve $20 \%$ moisture concentration was calculated and added in the jar. By assuming linear rate of hydration, given finite volume and water we were able to calibrate the amount of water needed for the sample to absorb moisture to a set level in 24 hours ( 48 hours to fully hydrate the higher moisture concentrated samples). The samples were removed from the oven after 24/48 hrs and weighed. The net sample mass and the moisture concentration was calculated before the transfer into neutron pressure cell.

\subsection{Pressure Cell}

Throughout the experiments we used a special high pressure cell, designed and built at the Queensland University of Technology (QUT), which allowed samples to be measured under independently controlled omni-directional fluid pressure up to $100 \mathrm{MPa}$ and an uni-axial mechanical pressure of up to $100 \mathrm{MPa}$ applied in the beam direction. The cell is ideally suited for this study, as it allows environmental isolation of the sample from the ambient environment and allows contrast matching $\mathrm{CD}_{4}$ to be used routinely. In addition, the cell offers the option of confinement to the sample as used in one measurement conducted in this study.

\subsection{Small Angle Neutron Scattering Measurements}

SANS measurements were performed on the NG-7 VSANS instrument located at the Gaithersburg MD campus of NIST. Neutrons that are produced in the 
reactor are slowed down and velocity selected to correspond to the wavelength of $5.5 \AA$ needed for these measurements. The beam then travels for $16 \mathrm{~m}$ before it is allowed to interact with the sample. The scattered neutrons are then collected by a $640 \times 640 \mathrm{~mm}$ detector operating under vacuum where its position can be altered, thus improving resolution. These measurements were taken at detector distances of $1 \mathrm{~m}, 4 \mathrm{~m} 13 \mathrm{~m}$ and a fourth position that used a set of neutron lenses effectively extending the detector's position further. All these results, once collected, were corrected for backgrounds and detector efficiency before being combined into a one consistent plot.

All measurements carried out on this instrument were done in the pressure cell described in Section 2.3. Figure 1 shows the cell at the SANS instrument. Mechanical strain was not altered during measurements, however gas was connected to the cell by a capillary, and an external pressure apparatus measured the internal pressure in real time.

Figure 2 shows the gas supply apparatus. Although capable of accurate gas mixing and gas sorption by the sample using the manometric method, for these measurements we only used it to keep the sample at a constant pressure. Although capable of accurate gas mixing and gas sorption by the sample using the manometric method, for these measurements we only used it to keep the sample at a constant pressure. Table 1 provides details of the samples used in the experiment.

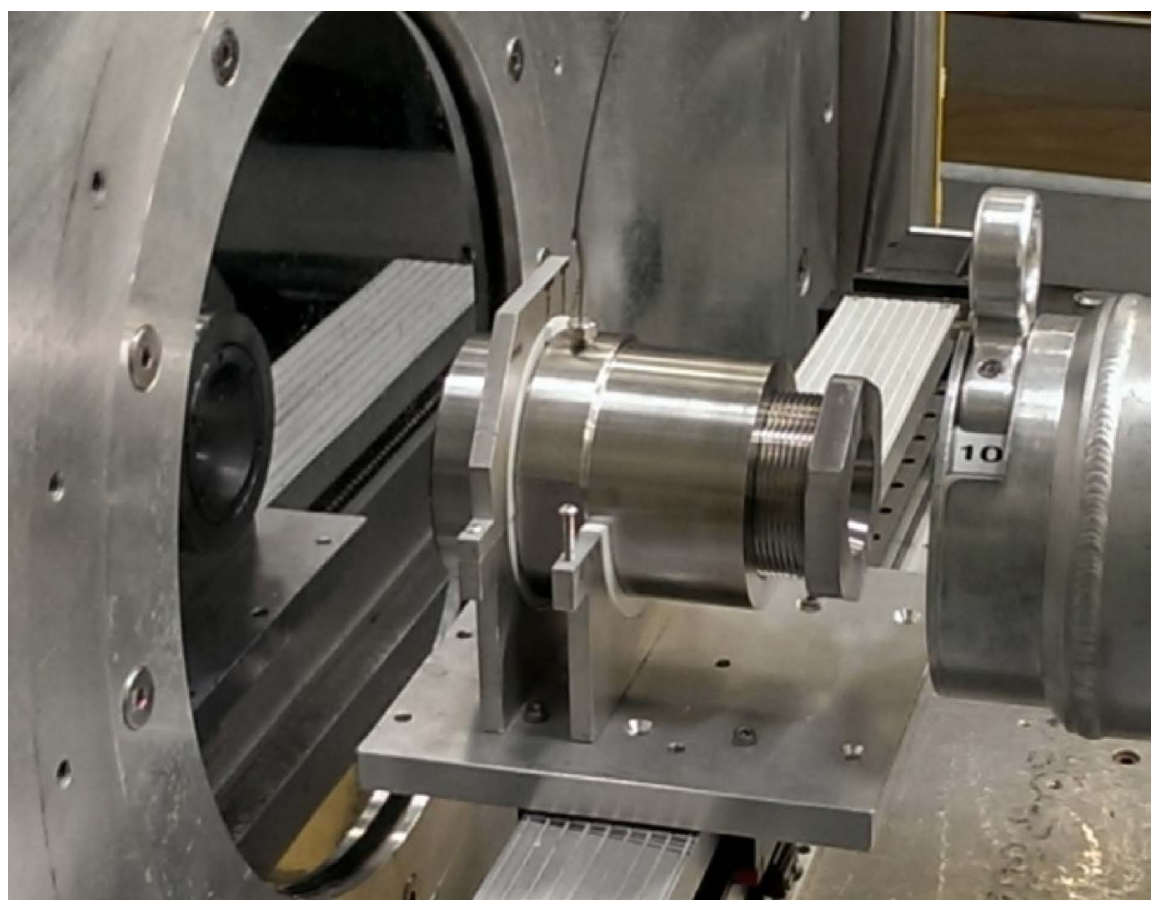

Figure 1. The high pressure SANS cell placed in the neutron beam in front of the detector tank. The neutron beam is reduced to a $10 \mathrm{~mm}$ diameter by an aperture and then enters the cell on the right hand side. Once it interacts with the sample, the scattered neutrons enter the detector tank on the left, via a high purity silicone window. Gas is introduced to the cell by the connecting $3 \mathrm{~mm}$ capillary visible at the top of the cell. 

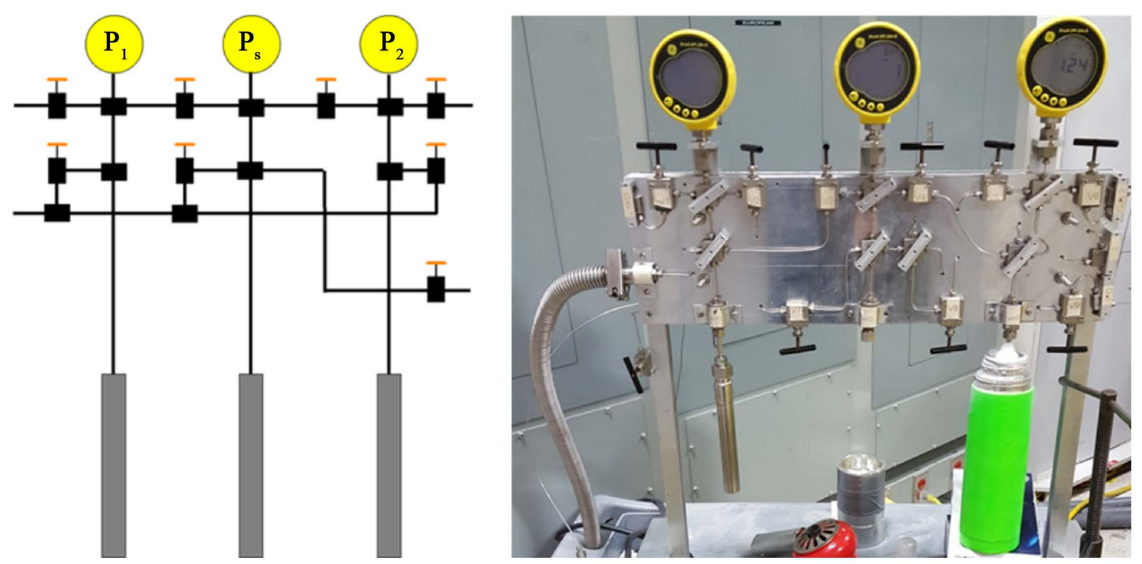

Figure 2. The gas handling apparatus schematic (left) and the working rig (right). $\mathrm{CD}_{4}$ required for these measurements were stored in the silver volume on the left. The gas was then transferred to the intensifying volume on the right, which was kept cold to liquefy the gas. Once pressure was needed, the cold thermos was removed and the volume was heated up using the red hot air blower until the required pressure was reached. Pressures in excess of $100 \mathrm{MPa} / 15,000$ psi could quickly be generated this way without the use of any moving parts.

Table 1. Samples used for SANS measurements.

\begin{tabular}{cccccc}
\hline Sample & Polymer & $\begin{array}{c}\text { Net mass } \\
(\mathrm{g})\end{array}$ & $\begin{array}{c}\text { Thickness } \\
(\mathrm{mm})\end{array}$ & $\begin{array}{c}\text { Moisture content } \\
(\text { weight\% })\end{array}$ & $\begin{array}{c}\mathrm{CD}_{4} \text { pressure used } \\
\text { at ZAC (MPa/psi) }\end{array}$ \\
\hline SA01 & - & 0.2863 & 0.91 & 0 & $48.3 / 7001$ \\
SA03 & - & 0.3273 & 1.03 & 18 & $48.4 / 7016$ \\
SA04 & - & 0.4647 & 1.22 & 33 & $48.4 / 7024$ \\
SP01 & + & 0.4182 & 1.28 & 0 & $48.4 / 7017$ \\
SP03 & + & 0.4525 & 1.19 & 18 & $48.5 / 7033$ \\
SP04 & + & 0.3672 & 1.11 & 33 & $48.4 / 7017$ \\
\hline
\end{tabular}

Based on the elemental composition of the bentonite plug material, as measured by X-Ray Fluorescence (XRF) (see Table 2) $S L D$ of the sample was calculated using [9] as 1.385. This value value includes the contribution of hydrogen expected to be present from the bentonite chemical formula. Adding a further $3 \%$ of the polymer into the elemental composition did not change the value of $S L D$ by more than $1 \%$ in value, thus for the rest of the experiment, we assumed the same $S L D$ for both polymer containing and polymer-free batches of samples.

As already mentioned, we hydrated our samples with the mixture of $\mathrm{H}_{2} \mathrm{O}$ and $\mathrm{D}_{2} \mathrm{O}$ so that the $S L D$ of the liquid and solid matched. Using [9] the water mixture needed was $\left(\mathrm{D}_{2} \mathrm{O}\right)_{0.54}\left(\mathrm{H}_{2} \mathrm{O}\right)_{1.46}$. This corresponded to the mass ratio of $\mathrm{D}_{2} \mathrm{O}$ to $\mathrm{H}_{2} \mathrm{O}$ of 1 to $2.265 \mathrm{~g}$. This ratio was then used to make approximately $100 \mathrm{~g}$ of the mixture used throughout these measurements.

The selection of this $S L D$ for the liquid was deliberate, as any pores or channels entered by this liquid would subtract from the data. This gives an indication if moisture pools in pores or cavities; however in the clay layers, there will be 
Table 2. Major element composition of the used bentonite from XRF measurements. As light elements such as $\mathrm{C}$ and $\mathrm{H}$ cannot be measured using this method, they were estimated based on accepted composition of bentonite.

\begin{tabular}{ccc}
\hline Composition & Element & Averaged atomic \% \\
\hline $\mathrm{Al}_{2} \mathrm{O}_{3}$ & $\mathrm{Al}$ & 7.48 \\
$\mathrm{CaO}$ & $\mathrm{Ca}$ & 0.90 \\
$\mathrm{Fe}_{2} \mathrm{O}_{3}$ & $\mathrm{Fe}$ & 1.30 \\
$\mathrm{~K}_{2} \mathrm{O}$ & $\mathrm{K}$ & 0.60 \\
$\mathrm{MgO}$ & $\mathrm{Mg}$ & 0.92 \\
$\mathrm{Na}_{2} \mathrm{O}$ & $\mathrm{Na}$ & 1.28 \\
$\mathrm{SO}_{3}$ & $\mathrm{~S}$ & 0.04 \\
$\mathrm{SiO}_{2}$ & $\mathrm{Si}$ & 21.26 \\
$\mathrm{TiO}_{2}$ & $\mathrm{Ti}$ & 0.24 \\
& $\mathrm{O}$ & 59.77 \\
\hline
\end{tabular}

excess of higher contrast water due to moisture already present there mixing with the new liquid. Any surface modification of the clay due to water would be detected by SANS at the interface between the clay and air present in the cell. The addition of $\mathrm{CD}_{4}$ at ZAC pressure would show signals left from any structures that cannot be accessed by either the water nor the gas-giving us information on the inaccessible content of this material.

The measurement session was started by collecting the dark current, the carbon glass standard and an empty pressure cell. The latter was measured with exposures $50 \%$ longer than the intended measurements with the samples. As the empty cell signal needs to be subtracted from all the sample measurement, it is critical to have the best statistics and signal to noise ratio in this measurement, as it will affect all the samples.

Following the calibrations, a ZAC confirmation experiment was performed. Sample SA01 was chosen for this, as it had minimal moisture content. The cell was loaded and closed. We then connected the cell to the gas handling apparatus via the $3 \mathrm{~mm}$ capillary and positioned the cell in the SANS instrument. We then positioned the detector at $4 \mathrm{~m}$ and intensity scan was collected for a minute. $\mathrm{CD}_{4}$ was then added in steps of $6.9 \mathrm{MPa} / 1000$ psi right up to $68.9 \mathrm{MPa} / 10,000 \mathrm{psi}$ and a 1 minute intensity scan taken with each step. A broad intensity minimum ranging from $34.5 \mathrm{MPa} / 5000 \mathrm{psi}$ to $62 \mathrm{MPa} / 9000 \mathrm{psi}$ was observed, confirming the expected ZAC pressure calculated from the compositional data. We thus decided to use $48.3 \mathrm{MPa} / 7000 \mathrm{psi}$ as the ZAC pressure for all the samples to be measured.

\subsection{Ultra Small Angle Neutron Scattering Measurements}

Following the SANS measurements we moved to the BT-5 USANS instrument. Figure 3 shows the schematic of the instrument. 


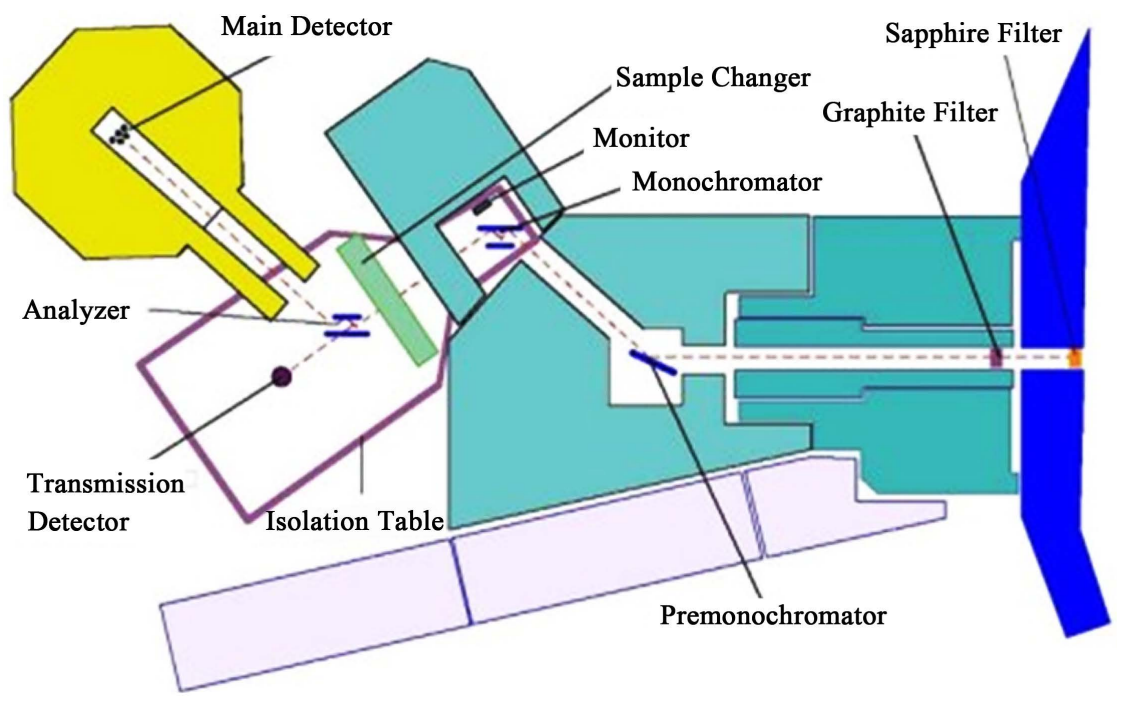

Figure 3. The BT-5 Perfect crystal USANS instrument. A beam of neutrons entering from the reactor on the right passes through crystal filters and reflectors to produce a monochromatic beam directed at the sample. The sample is placed between the monochromators and an analyser crystal, both made to a very high level of precision. The analyser crystal rotates slightly, thus allowing the angle to be selected for the detector to count. The instrument is thus compact, but slow due to reduced flux from all the filtering. Furthermore, it uses slit geometry so samples should be isotropic.

As we were unable to maintain the moisture of each sample stable once the SANS data was collected we decided to prepare a fresh batch of samples for the USANS measurements. Furthermore, since we had more beam time than needed for the measurement of the six SANS samples, we prepared extra samples to get some idea of any trends that emerge at additional moisture concentrations. All samples measured on BT-5 are summarised in Table 3.

Samples SAS1 and SPS1 were saturated in a different way to all the other samples. Instead of using the hydrator, we applied water droplets directly onto the sample. This was done on a laboratory balance, so that the mass gain was measured in real time. Once the right weight was reached, the sample was quickly transferred to the pressure cell and allowed to reach equilibrium for about $1 \mathrm{hr}$.

\section{Results}

Each SANS measurement used a beam of thermal neutrons, with a wavelength of $1.498 \AA$ and beam diameter of $15 \mathrm{~mm}$ aimed at the cell and collimated down to $10 \mathrm{~mm}$ at the sample. While passing through the sample, the neutrons scattered and travelled to the detector. The neutrons were collected by a 2D neutron detector positioned at a selected distance from the sample and the rate of neutrons per unit time as a function of detector position was measured. Figure 4 shows a typical raw detector output, converted to a color plot.

The raw data collected by the instrument detector has to be processed before it can be used to extract the information of interest. The aim of the technique is to measure the absolute intensity profile of each sample, that is the scattering 


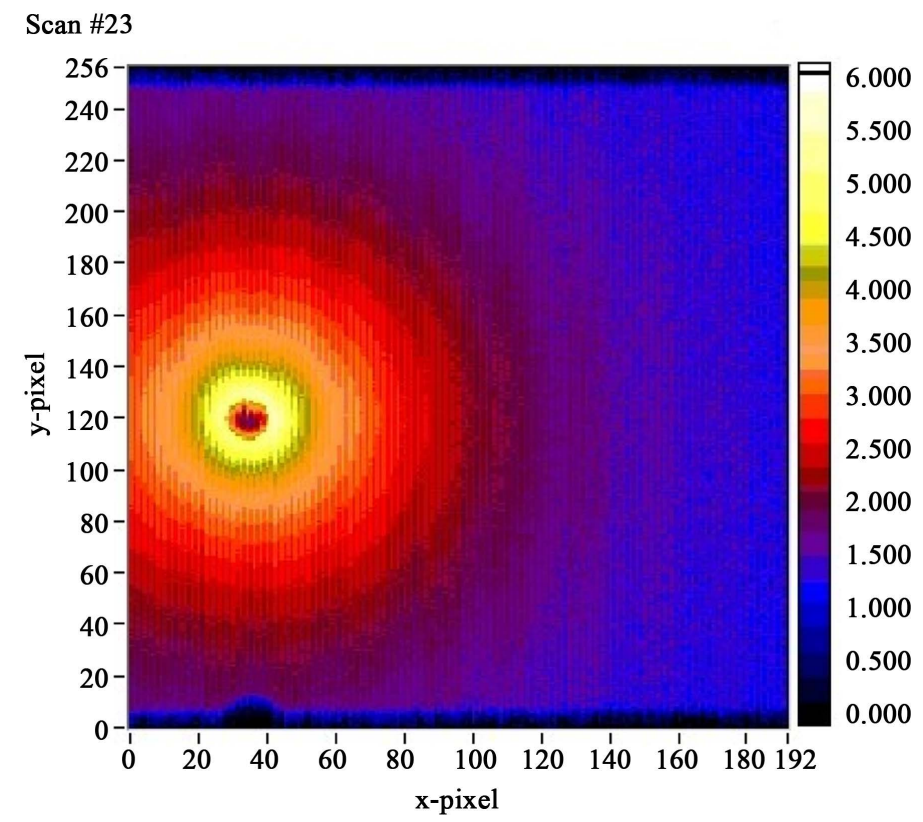

Figure 4. A raw intensity plot collected from the NG7 SANS instrument at NIST. To prevent saturation of the detector from the unscattered beam of neutrons, the centre of the circular pattern (thus the unscattered remnants of the beam) is masked. The image is converted to the absolute intensity $[I(Q)]$ vs $Q$ (equivalent to radial position) by the application of data correction from calibrations and de-smearing procedures.

Table 3. Samples measured using the BT-5 USANS instrument. Samples SA05 and SP05 are measured twice: the first in a standard configuration and the second time internally strained by 6 tonne uniaxial strain, imitating confinement.

\begin{tabular}{cccccc}
\hline sample & polymer & $\begin{array}{c}\text { net mass } \\
(\mathrm{g})\end{array}$ & $\begin{array}{c}\text { thickness } \\
(\mathrm{mm})\end{array}$ & $\begin{array}{c}\text { moisture content } \\
\text { (weight\%) }\end{array}$ & $\begin{array}{c}\mathrm{CD}_{4} \text { pressure used } \\
\text { at ZAC }(\mathrm{MPa} / \mathrm{psi})\end{array}$ \\
\hline SA01 & - & 0.2863 & 0.91 & 0 & $48.2 / 6997$ \\
SA03 & - & 0.4415 & 1.17 & 17 & $48.3 / 7013$ \\
SA04 & - & 0.3801 & 1.21 & 33 & $48.2 / 6997$ \\
SA05 & - & 0.4148 & 1.20 & 9 & $48.6 / 7053$ \\
SA05 & - & 0.4148 & 1.20 & 9 & $48.4 / 7027+6 \mathrm{t}$ conf \\
SAS1 & - & 0.4594 & 1.43 & 46 & $48.4 / 7020$ \\
SP01 & + & 0.4182 & 1.28 & 0 & $48.4 / 7022$ \\
SP03 & + & 0.4373 & 1.21 & 19 & $48.4 / 7018$ \\
SP04 & + & 0.4515 & 1.30 & 37 & $48.6 / 7044$ \\
SP05 & + & 0.3943 & 1.00 & 8 & $48.5 / 7032$ \\
SP05 & + & 0.3943 & 1.00 & 8 & $48.4 / 7016+6 \mathrm{t}$ conf \\
SPS1 & + & 0.2881 & 0.97 & 43 & $48.5 / 7031$ \\
\hline
\end{tabular}

intensity, $[I(Q)]$, as a function of the scattering vector $Q$ (or roughly the size of the scatterer). Ideally, this would be measured as if the scattering originated from a single particle without any smearing from adjoining particles. In practice 
this is impossible, and it is expected that the contributions from the different scatters will interfere with each other and the smearing of the intensity data would result. In addition, the raw data must be corrected for the various parasitic contributions to the signal: the contribution from the sample cell, detector efficiency at different camera lengths, beam intensity fluctuations, etc. Thus by collecting data from the empty cell and various standards, appropriate corrections can be measured and applied. With the aid of these, the 2D intensity pattern can be converted into the absolute intensity $[I(Q)]$ as a function of scattering vector $(Q)$ plot for parameter extraction.

These corrections were applied to our raw data using the NIST's IGOR-PRO SANS data processing package which applies these corrections to the circular 2D pattern, based on the data collected during the calibration phase. USANS on the other hand uses slit geometry to collect data, so the processing is done by a different IGOR PRO package. The NIST USANS data processing package was used to correct the raw intensity for the contributions of the empty cell, and the instrumental errors. To obtain the absolute intensity, it is assumed that the sample is perfectly isotropic and the equivalent pin-hole pattern is circular, which was the case with these samples as seen in SANS patterns. These Intensity plots are then de-smeared numerically by the NIST USANS processing package and an absolute intensity pattern is recreated that can be spliced to the existing SANS profile, extending the spatial range to micrometres.

\subsection{Combined SANS and USANS Measurements}

The first part of the measurements involved the raw bentonite and the polymer-bentonite blend samples with a progressive hydration. These samples were summarised in Table 1 and Table 3 depending on which instrument was used. Figure 5 shows the absolute intensity plots over the full spatial range for these samples. The data reported in Figure 5 has a linear trend over most of the $Q$-range. The curves flattened at high $Q$ s (the region where the smallest scatterers contribute) by the incoherent scattering and a contribution from Bragg scattering.

The application of moisture appears to affect the high $Q$-range (small scatterers) immediately, filling these pores (or cavities) up and preventing both the gas and extra water from creating more of them. These structures lie in the $Q$-range above 0.01 (less than $60 \mathrm{~nm}$ size) and thus appear to respond the fastest to even the small amounts of moisture. There appears to be a small Bragg's peak present in wet samples at $Q \sim 0.5^{\mathrm{A}-1}$ most likely due to the laminar layers in the clay and a further broader peak at about $Q \sim 0.013^{\mathrm{A}-1}$, due to some other structure present in the clay. The addition of the polymer binder appears to have a significant effect on the nature of the larger structures in the plug material, as shown by the greater variation in the slope and intensity at a given $Q$ in the mid to small $Q$-range. This is not so obvious in the raw bentonite, suggesting that the addition of extra water plays little effect on the larger structures formed by the clay particulates. 

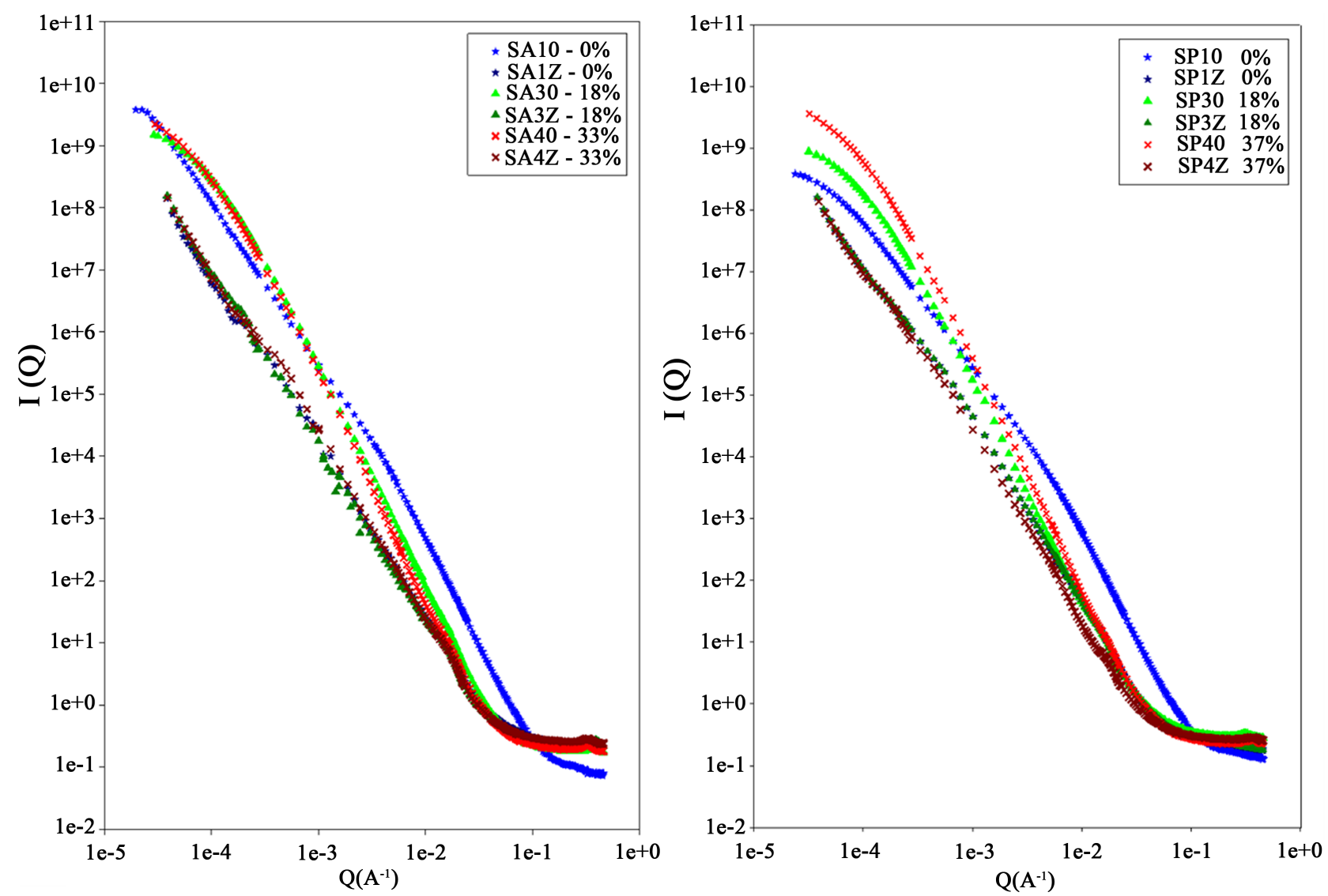

Figure 5. Combined absolute intensity plots for the reportedsamples. Left: raw bentonite, Right: bentonite-polymer blend.

Again, in the USANS Q-range (about $300 \mathrm{~nm}-20 \mu \mathrm{m}$ ), the presence of the polymer has the most effect on the intensity plot. Although this region is typically most affected by the parasitic effects of multiple scattering (which cannot be modelled mathematically), the fact that the curves do not change shape in the gas saturated measurements suggests that this effect is small. Whereas the presence of moisture has practically no effect on the intensity curves of the not gas treated sample, the presence of the polymer increases the scattering with moisture content, suggesting that some open structures are mediated by the polymer chains. These structures are open and when the ZAC condition is applied, they disappear as the ZAC signal does not change with moisture content.

\subsection{USANS Measurements}

As already stated previously, more samples were measured on the BT-5 instrument (USANS) than on the SANS machine. Although the additional data has no useful information on the high $Q$-region (small scatterers) observation in the micrometer range was made. Figure 6 shows the data collected on the raw bentonite plug material and Figure 7 on the polymerized samples.

In contrast to the SANS data, it appears that the addition of moisture creates structures in the clay that are open to the methane. In fact over the entire $Q$-range investigated by the technique, there is a drop in intensity of nearly $90 \%$ 

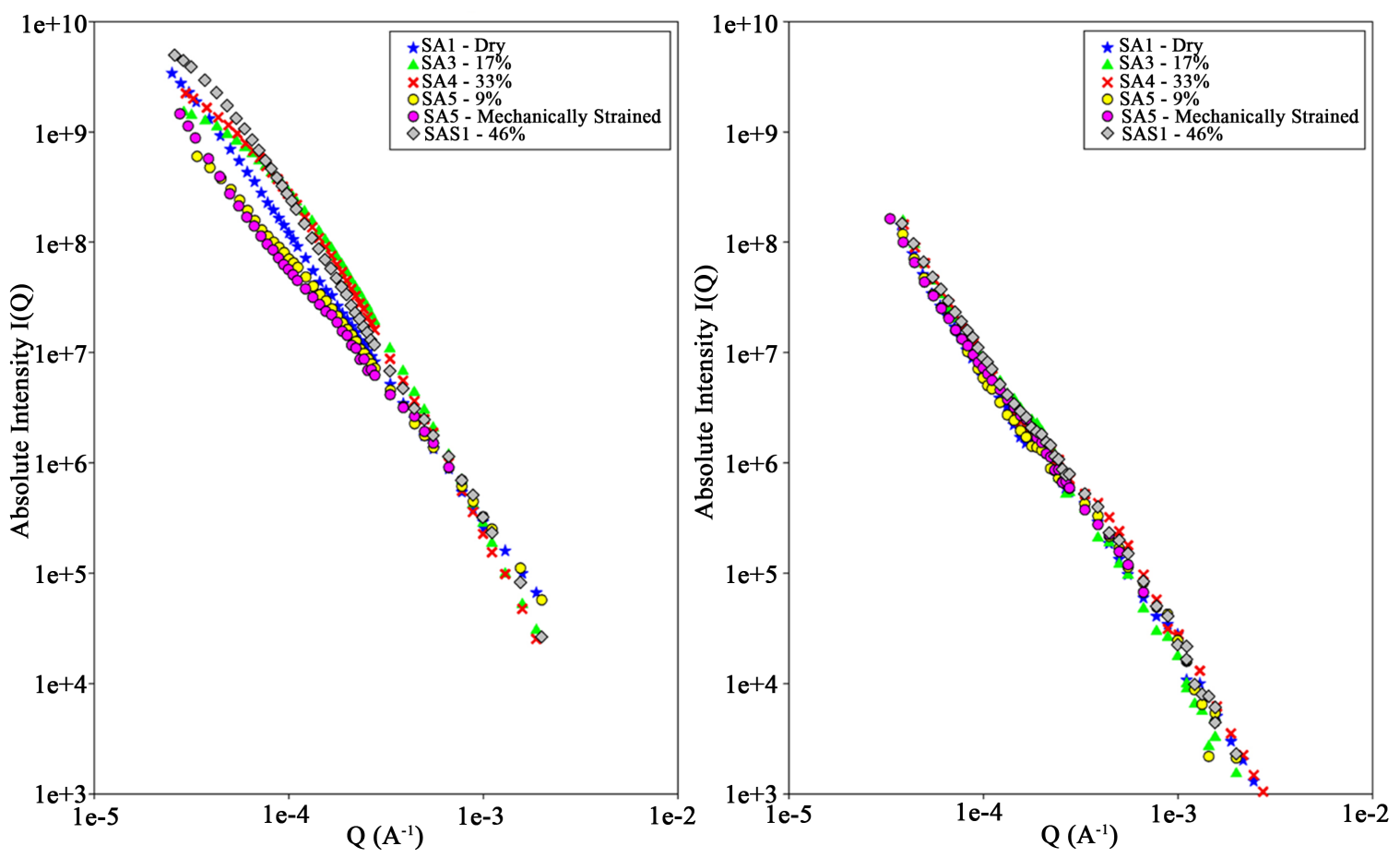

Figure 6. USANS absolute intensity plots for the six untreated bentonite samples reported in Table 3. Left: raw bentonite at no gas pressure; right: raw bentonite at ZAC gas pressure. The data shows that although the gas affects the entire range by reducing the scattering intensity, moisture alone affects the low- $Q$ or sub-micron region the most.
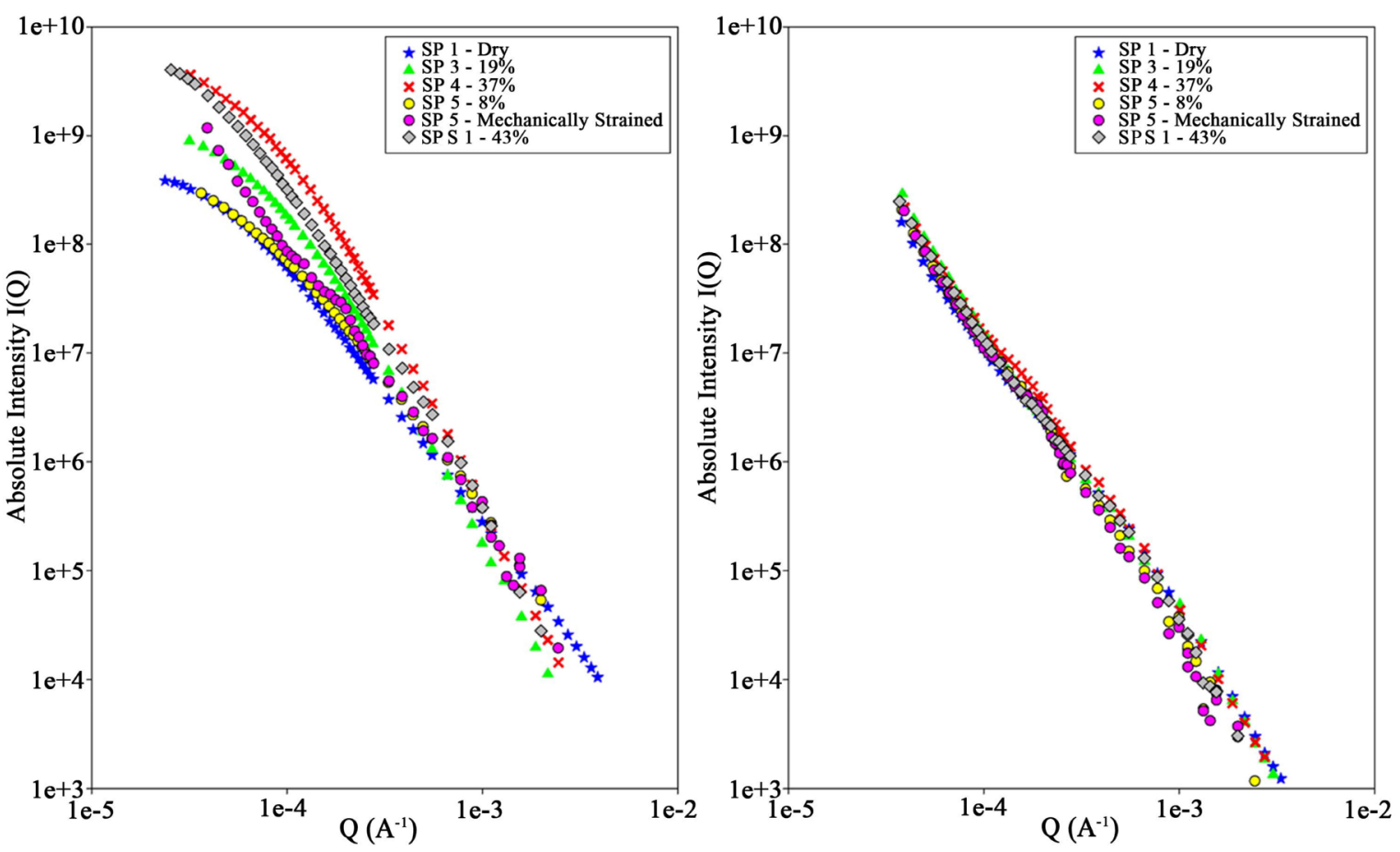

Figure 7. USANS absolute intensity plots for the six polymerised bentonite samples reported in Table 3. Left: polymerised bentonite at no gas pressure; right: polymerised bentonite at ZAC gas pressure. The data shows that although only about $1 \mathrm{wt} \%$ of polymer were added to stabilise the clay, this has the biggest impact on the micron size scatterers, as the addition of moisture increases the scattering in that region by over 10 times. Again, as with the untreated bentonite, once fully saturated by gas, little difference in scattering intensity as a function of moisture content is observed. 
with the introduction of the gas, but only a moderate drop, or no drop in intensity over regions in the $1 \mu \mathrm{m}$ range when moisture is added. As already noted, it would appear that the largest structures are mostly affected by the inclusion of the water, however the addition of the polymer amplifies the effect.

In the raw bentonite sample, it appears that the addition of a small amount of water ( $9 \%$ by weight) reduces contributions from the large regions, suggesting that the signal comes from regions that act as pores and the $S L D$ of this water removes them. When mechanical strain was applied on this sample, there was practically no effect on the signal, except for a region in the $Q \sim 1 \times 10^{-4} \AA^{-1}$, where it fell a little more. This suggests that the extra strain played little role in the initial hydrogenation of the sample. Due to limited beam time, we could not however investigate if this is the case at higher concentrations and it remains to be tested.

There is limited evidence that in the upper regions of the SANS-range that overlaps with the lower ranges of USANS the slope of the power law decreases with the added moisture, however as the statistics in this range are worse in the USANS results, this must be confirmed with measurements on SANS in the future.

Saturating the sample with liquid water as opposed to water vapour appears to have a very limited effect in the USANS-range, affecting only the biggest structures sampled. It appears that more of these are formed-suggesting that some particulates segregated from the plug material in the presence of "liquid" water (the increase in slope suggests more profounded mass fractality), however as the contribution of multiple scattering cannot be excluded, these results should be confirmed with additional measurements.

At ZAC condition, the moisture does not appear to affect the sample at all, indicating that what is seen is a contribution of closed pores and non-bentonite impurities and these participate very little in the hydration of the sample. This signal accounts only for about $10 \%$ of the USANS data.

Addition of the polymer binder to the plug material changes the plugs USANS intensity plot in a different way. Figure 7 shows that this time the whole spatial region responded. Again the biggest effect occurs at the largest structures investigated (small $Q$-region) and the number of these scatterers increased systematically with the water concentration.

Addition of small quantities of vapour (about $8 \mathrm{wt} \%$ this time) appears to have a very small effect on the material in this $Q$-range. What is of great surprise however is the application of the uniaxial strains on this sample, as in the smallest $Q$-range this had a very significant effect, changing the shape of the intensity curve. This result suggests that there is little multiple scattering affecting this result and that the structures are fractal beyond the resolution of USANS. The compression creates these open structures in the smallest $Q$-range whilst affecting the scattering in the bigger $Q$-range very little. Again there is no SANS data to see the effect on the nano-structure, but the result suggests that under strain, the polymer may form hollow structures that form larger cavities that are pene- 
trated by the gas. Water penetration is less and may be due to the water being locked up in the finer structures instead.

Also of interest is the behaviour of the sample saturated with liquid water. While this action produced the highest intensity in the low $Q$-range of any of the clays, this is not the case in the polymerised sample, suggesting that the vapour in this case produces more of the scatterers than exposure to the liquid.

\section{Discussion}

The results presented above must be understood in terms of the clay properties already known from other studies, as well as by a supporting interpretation of the data presented in the previous section. Thus the nature of the bentonite in combination with the technique will be discussed first, before a full picture revealed by this study is presented.

\subsection{Properties of Bentonite}

Sodium or calcium bentonite is the name given to a material whose major constituent is the mineral montmorillonite. The montmorillonite mineral belongs to the smectite mineral group, in which all members have an articulated layer structure and swelling properties. They consist of a three-layer structure that is made up of two tetrahedral layers sandwiched around a central octahedral layer (see Figure 8).

Oxide anions at the apices of the tetrahedral subunits are directed inward where they surround interior aluminium, iron, and magnesium cations, thereby forming the octahedral subunits of the octahedral layer. Bonding between the shared interior oxide anions and cations, in both the tetrahedral and the octahedral layers, links the layers together and yields the unique sheet structure characteristic of clay mineral. Minerals of this type are often referred to as 2:1 layer structures [10].

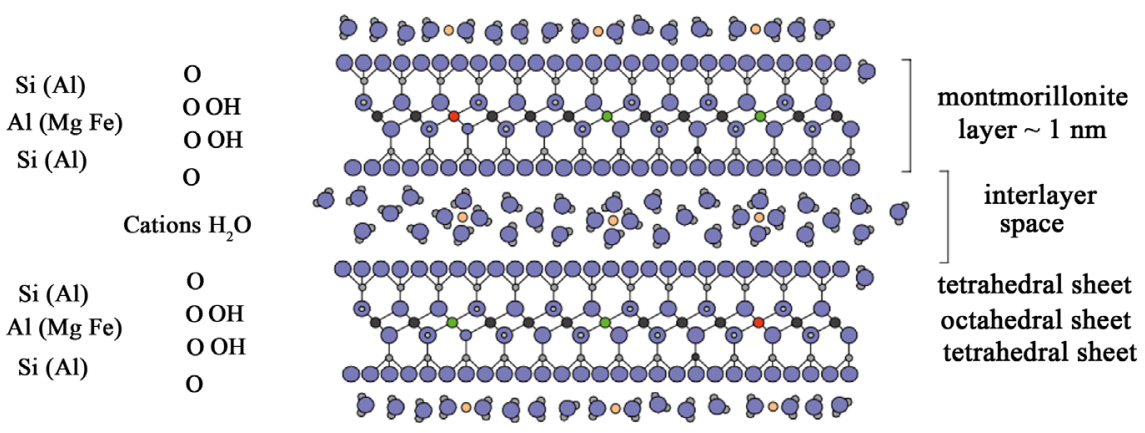

Figure 8. The "on edge" representation of a smectite clay that forms the basis of the bentonite mineral. The mineral forms montmorillonite layers (about $1 \mathrm{~nm}$ thick) that extend for hundreds of nanometres in a plane perpendicular to this edge. These layers are separated by the interlayer space, which fills with water during hydration and produces the swelling effect. Depending on the concentration of water molecules in this interlayer, the water molecules form a concentration-dependent layering effect. This is the basis of bentonite swelling (Figure reproduced from [10]). 
For montmorillonite, the total negative charge contributed to the structure by the sum of all the oxide anions $\left(\mathrm{O}^{-}\right)$is somewhat in excess of the total positive charge contributed by the sum of all the structural cations $\left(\mathrm{Si}^{4+}, \mathrm{Al}^{3+}, \mathrm{Fe}^{2+}, \mathrm{Fe}^{3+}\right.$, $\mathrm{Mg}^{2+}$ ), and imparts a slight overall negative charge to the surfaces of the clay sheets. This slight excess negative charge on the sheets is counterbalanced by free-moving (exchangeable) cations, which exist between them. These three layers in each sheet comprise individual bentonite platelets, which are typically 1 $\mathrm{nm}$ in thickness and may be over $100 \mathrm{~nm}$ in diameter. Typically, these platelets are grouped together in a face-to-face arrangement, with exchangeable cations and small amounts of absorbed water in an interlayer region between each platelet. The thickness of the interlayer region is variable depending on the amount of water absorbed between the platelets [11].

The remaining part of the bentonite may vary substantially in mineralogy within, and especially between different quarries, and depends on the geochemical conditions during the formation of the bentonite. Typical accessory minerals present in bentonites are other clay minerals, quartz, feldspars, gypsum, calcite, pyrite and various iron oxides/hydroxides. Additionally, amorphous and organic compounds not easily detectable by X-Ray diffraction analysis may be present in significant amounts, adding to the complexity of the material [10].

Bentonites are generally classified according to their dominant exchangeable interlayer cation, usually sodium or calcium, although magnesium may also be present. When sodium predominates, a large amount of water can be absorbed in the interlayer, resulting in the remarkable swelling properties observed with hydrating sodium bentonite. The presence of sodium facilitates the almost unlimited absorption of oriented layers of water molecules, a phenomenon which does not occur when calcium is predominate. Consequently, calcium bentonites are generally lower swelling [11].

As already mentioned, upon hydration bentonite is expected to swell, the process being driven by the accumulation and ordering of the water molecules in the interlayer space. Since water is a molecule with strong polarity, as it enters the montmorillonite interlayer space, the hexagonal oxygen surface of the clay makes an attractive adhesion region for the hydrogen atoms of the water molecules, so the water molecule orients preferentially forming a water layer. Moreover, as there is a presence of excess electrons on the surface of each montmorillonite layer, these electrons enable the formation of enhanced hydrogen bonds of partially covalent character between the water molecules and the surface. The result of this is not only the strengthening of the bentonite-water bond but also the facilitation the bonding of additional layers of water. It is believed that these strong bentonite-water bonds alter the electron distribution of the first layer of water molecules and facilitate the formation of another water layer. Those bonds in the next layer allow the formation of a subsequent layer to the extent that 3 or 4 similarly oriented water layers are formed. However, the kinetic nature of water causes the strength of the orienting forces to decrease with distance, and (if enough free water is present), the additional layers of water molecules become 
increasingly less rigid as the distance from the bentonite surface increases [12].

The negative charge and hexagonal arraignment of oxygen atoms on the bentonite surface facilitate the formation of the first few oriented water layers. Thereafter, it is generally accepted that the presence of sodium ions produces osmotic pressure, which draws water molecules into the interlayer, in an attempt to equalise the high concentrations of ions between platelets with the low concentrations of ions outside the platelets [12]. The result is an increase in the interlayer pressure and a swelling of the clay [13].

In the industry, the degree of the swelling of the bentonite is a measure of performance [11]. The more the clay swells, the better it seals and heals any cracks. In a typical bentonite aggregate, hydrated platelets are aggregated by face to face and edge to face electrostatic bonding (see Figure 9 (left)) with perhaps only one or two oriented water monolayers between adjacent sheets. This limits the kinetics and the swelling effect of the clay, thus reducing its performance. In response, manufacturers of bentonite products have experimented with the additions of polymers to the clay mixture in order to improve water access to the interlayers and to minimise the electrostatic bonding between interlayers. Figure 9 (right) shows how the polymers are believed to help the bentonite to form more open aggregates.

SANS and USANS are techniques ideally suited to investigate the physical structure of the clay material, as they provide structural information from the sub-nano to micro-metre length scales. The addition of polymer and the effect of compaction on the specific plug materials are reported below.

\subsection{Small Angle Scattering Principles}

Subatomic particles, such as neutrons and photons, are used to probe matter. As these particles are typically smaller than the atom, they are quantum in nature, meaning that they behave as both particles and waves when they interact with matter and each other. When the energy of the particle is such that its wavelength corresponds to the interatomic distances, the particle will scatter elastically off the atoms in the sample. As X-Rays scatter off the electrons, they scatter weakly off of the light elements, such as hydrogen, and strongly off the atoms
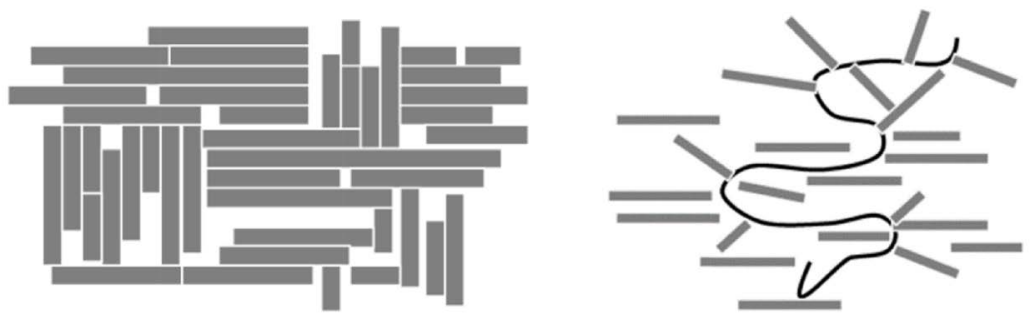

Figure 9. Simplistic representation of the edges of the platelets of dry bentonite in 2D space. In the pure, dry form (left), it is believed that the edge to edge and surface to edge bonding limits the clays swelling performance. Addition of polymers to the mixture, re-orients the platelets, thus increasing the surface area available to water thus improving hydration performance (right) [11]. 
with many electrons. Neutrons, on the other hand, carry no electric charge and instead scatter off of the atomic nuclei. This gives them a unique ability to distinguish between different isotopes of the same element and the strength of the scattering depends on the nuclear arrangement of the atoms being probed [14].

The Scattering Length Density $(S L D)$ is a measure of the elastic scattering power of a material and for neutrons it can have either a positive or negative value. For example a material composed of only hydrogen (whose nucleus is only made of the proton) has a $S L D$ of $-1.5 \times 10^{-6} \AA^{-1}$, while the same material made from the isotope of hydrogen, known as deuterium (whose nucleus is composed of a proton and a neutron) has a $S L D$ of $2.823 \times 10^{-6} \AA^{-1}$. As both hydrogen and deuterium are chemically identical, it is possible to generate materials with different ratios of these isotopes such that the mixture can have a fine tuned $S L D$. Since a beam of neutrons or X-Rays scatters off of the interface between regions of different $S L D$, known as contrast, samples can be modified isotopically to enhance or diminish contrast as needed for the application.

In the Small Angle Scattering (SAS) regime, the beam scatters off domains with different contrast to that of the surrounding matrix. These domains can be either particles in a solvent or pores distributed in a matrix. Thus, the aim of the measurement is to collect the intensity function $I(Q)$ (that is the number of neutrons arriving at the detector in a unit of time) at a specific solid angle away from the beam axis (given by the scattering vector $Q$ ) as:

$$
I(Q)=V_{0} \overline{\Delta \rho^{2}} S(Q)
$$

where $V_{0}$ is a constant in the order of the average pore volume and incorporates instrumental calibration parameters; $S(Q)$ is the structure factor function for a diluted system of scatterers, which contain the geometrical information of the sample measured; and $\overline{\Delta \rho^{2}}$ is the scattering contrast, defined in terms of the difference in $S L D$ 's between the two media as:

$$
\overline{\Delta \rho^{2}}=\left(S L D_{1}-S L D_{2}\right)^{2} \phi(1-\phi)
$$

where $\phi$ is the volume fraction of the particle space.

Information related to the quantity, shape and size of the scatterers can be extracted from the Intensity function and with the use of carefully selected medium, much can be learned about the system under study.

Radlinski et al. [15] [16] has shown that for a fractal sample with size-independent contrast, we would expect a relationship between the intensity and the scattering vector to obey a Power Law.

$$
I(Q)=K Q^{-D}
$$

where $K$ is a constant and $D$ represents the fractal dimension of the sample.

Since the neutron beam diameter is large when compared to the size of the scatterers and the $S L D$ of most of the compositional components of the sample are similar in magnitude, we can approximate the system as being essentially two-medium system, composed of clay matter and the suspension fluid (air). 
Moreover, as we were interested to learn how the physical structure of the plug material changes with hydration, we have decided to use a $\mathrm{H}_{2} \mathrm{O} / \mathrm{D}_{2} \mathrm{O}$ mixture that matched the $S L D$ of the clay. This makes the space available to the water (pores) "disappear" from our scattering profiles as moisture is added. Thus we can track the structures (or layers, pores etc.) that are most preferred by the moisture as a function of water content.

The power law described by Equation (3) is encountered in most sedimentary rocks [14] [16]. Yoonessi et al. [17] have shown that this also holds true for any clay material and that changing moisture and polymer concentration does affect the power index $\mathrm{D}$ in the intensity function. They were also able to determine the average size of the clay platelets based on the $Q$ dependent changes in $\mathrm{D}$. In this work, we explored this further, by also allowing $\mathrm{CD}_{4}$ at $\mathrm{ZAC}$ pressure to penetrate the sample fully and effectively switch off any accessible pore (or channel) network that still existed when the sample was modified by the extra moisture.

\subsection{Kratky Analysis}

In the high $Q$-region (the smallest scatterer region), the intensity is low and thus incoherent scattering from the hydrogen atoms is significant. The incoherent background is typically subtracted from the data and the data is plotted as a plot of Intensity multiplied by $Q^{2}$ vs $Q$. The resulting plot, known as the Kratky plot, emphasises any non-linear deviation from the high- $Q$ behaviour of the scattering intensity. It is typically used to measure the underlying nature of the material being studied to distinguish it between a mass fractal and a polymer-like chain structure for example. In our case, it also is used to "amplify" any Bragg reflections that are expected to arise from the underlying montmorillonite structure making up the sample [14].

Figure 10 shows the enhanced peaks, with background subtracted. These peaks were fitted with a Gaussian function to pin-point the central position and a relative height. Table 4 summarises the findings. Both dry clays (SA01 and SP01) show the same average platelet spacing of $22.7 \pm 0.1 \AA$ when dried. This is consistent with results reported by Yoonessi et al. [17] on dry, organically and mechanically modified clays. It shows that treatment with the polymer does not affect this spacing and suggests that this is specific to this material and its unique processing to form the plugging material. The low intensity and significant broadening of the peak suggests that these structures are not homogenous and are strongly deformed. Again this is consistent with a hard, dry bentonite, a result of mechanical deformation during the plug manufacturing process and vacuum drying [18]. Application of methane at ZAC pressure to these samples shows, however, that these structures are significantly affected by the presence of the gas and contrary to expectation, the size of these peaks significantly increased.

Bentonite is believed to be made up of aggregates and clusters containing pores and channels which direct the water molecules during hydration to the 

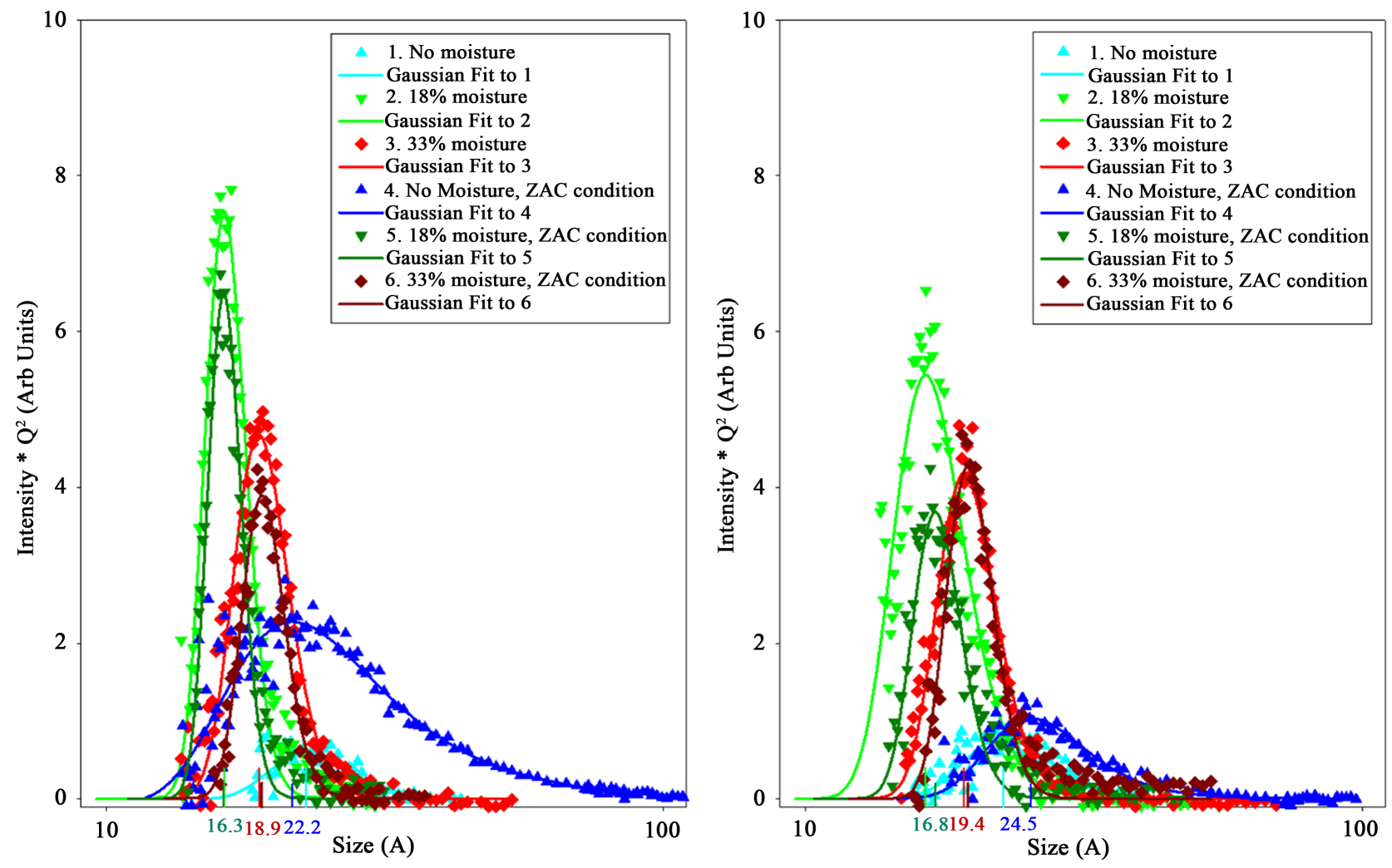

Figure 10. Kratky plots were used to enhance and to probe the data in the high Q-region of the intensity plotwhen incoherent scattering was subtracted(raw bentonite: left; polymerised bentonite: right). A peak corresponding to a Bragg reflection was observed and its intensity and breadth changed with each sample. The peak from dry bentonite samples indicate some periodic reflecting planes in the material separated by $22.7 \AA$. Application of methane at ZAC pressure altered this spacing as position and intensity of these reflections were affected (blue curves). This was sensed the most by the raw sample (left), where the intensity increased with the addition of the gas. Upon uptake of moisture by both materials, the interlayer spacing changed and well defined reflections formed corresponding to $16.3 \AA$ (green) and $18.9 \AA$ (red) interlayer spacing with 18 wt\% and 33 wt $\%$ water uptake respectively.

Table 4. Values corresponding to the Gaussian peak fit in the Kratky plot.

\begin{tabular}{ccccc}
\hline Sample & Added water (wt\%) & $\begin{array}{c}\text { Peak height } \\
\text { (arb units) }\end{array}$ & $\begin{array}{c}\text { Peak position } \\
(\AA)\end{array}$ & $\begin{array}{c}\text { Peak width } \\
(\AA)\end{array}$ \\
\hline SA01 & 0 & 0.585 & 22.8 & 3.83 \\
SA01 $+\mathrm{CD}_{4}$ & 0 & 2.34 & 21.6 & 6.94 \\
$\mathrm{SA03}$ & 18 & 7.55 & 16.3 & 1.36 \\
$\mathrm{SA} 03+\mathrm{CD}_{4}$ & 18 & 6.50 & 16.2 & 1.11 \\
$\mathrm{SA04}$ & 33 & 4.68 & 18.8 & 2.10 \\
$\mathrm{SA04}+\mathrm{CD}_{4}$ & 33 & 3.89 & 19.0 & 1.67 \\
$\mathrm{SP} 01$ & 0 & 0.763 & 22.6 & 3.99 \\
$\mathrm{SP} 01+\mathrm{CD}_{4}$ & 0 & 1.03 & 25.4 & 5.17 \\
$\mathrm{SP} 03$ & 18 & 5.45 & 16.4 & 2.38 \\
$\mathrm{SP} 03+\mathrm{CD}_{4}$ & 18 & 3.69 & 17.1 & 1.69 \\
$\mathrm{SP} 04$ & 33 & 4.19 & 19.3 & 2.31 \\
$\mathrm{SP} 04+\mathrm{CD}_{4}$ & 33 & 4.28 & 19.6 & 1.87 \\
\hline
\end{tabular}


montmorillonite interlayers. The process of water diffusion is believed to occur through two distinct processes-capillary suction in the channel and ionic attraction into the interlamellar space. As the arrangement of the water molecules in the montmorillonite is strictly governed by the shape and electrical properties of the water molecules and the electrical charges on the basal surfaces, it is very unlikely that methane would diffuse through these layers. Instead, Push argues that gas movement in wet clays takes place by firstly penetration of the microchannels and then by the displacement of soft gels in them [18]. This then implies that the influence of the gas on the clay would be greatest in the void and channel space and not in the interlayer, thus any changes in the Bragg peak due to the presence of the gas are then expected to originate from the rearrangement of the water-clay structure.

Introduction of the high pressure methane into the plug material had an immediate effect on the shape and size of these peaks. While the intensity of both samples increased with the added gas, the peaks of these two materials shifted in the opposite directions in the $Q$-space, that is the peak shift in the polymerised sample experienced a $6 \%$ contraction, while the polymerised sample separation swelled by $11 \%$. This result may indicate that although these clays had the minimum concentration of water and as such should have the smallest montmorillonite layer separation, the combination of impurities and mechanical processing created a semi-stable physical structure with a larger basal spacing. Whilst the isobaric pressure brought in by the introduction of the methane compressed these structures in the raw bentonite, it seem that the methane impacted the polymer in such a way as to actually increase both the interlayer spacing and its size distribution indicated by the doubling of the peak width.

Addition of moisture to the plug material improves the homogeneity of the clay layers as evidenced by sharper and bigger peaks. The larger interlayer separations measured in the dry material, was replaced by peaks corresponding to an interlayer spacing of $16.3 \pm 0.1 \AA$ when hydrated to $18 \mathrm{wt} \%$. However, whilst there is no noticeable peak shift in the raw material when methane was added, the polymerised material experienced a further interlayer swelling of about $5 \%$ in the presence of methane. Despite the use of contrast matched water mixture, the intensity of the diffraction peak has actually increased significantly, but fell as expected with the addition of methane. This suggests that the $\mathrm{H}_{2} \mathrm{O} / \mathrm{D}_{2} \mathrm{O}$ mixture actually present in these interlayers is different to the one introduced during hydration. This is expected, as the monolayer of water expected to exist in the dry sample would have been nearly totally composed of hydrogen, thus resulting in a mixture different to that required to remove contrast. The narrowing of the peak also suggests a more homogenous structure, pointing out to the physical re-arrangement of aggregates and laminar structures during hydration.

Compression with $\mathrm{CD}_{4}$ had a relatively small effect on the un-polymerised sample, but surprisingly it helped to swell the layers by an extra $5 \%$ in the polymerised sample. This may be due to the polymer affecting ion flow from the impurities present in the bentonite into the water filled spaces, as it is well known 
for the swelling to be strongly affected by the concentration of $\mathrm{Na}^{+}$and $\mathrm{Ca}^{2+}$ ions [19]. The peak was also much wider in the polymerised equivalent sample indicating more inhomogeneity in the sample, consistent with the assumption that the polymer affects the ionic flow in the material. The exact nature of this can be investigated with further studies, in particular using deuterated polymers and the analysis of the incoherent scattering component of the SANS data. Reduction in the scattering intensity in the presence of methane is consistent with $\mathrm{CD}_{4}$ entering accessible pores and channels, reducing their contribution to the total scattering at that $Q$.

Further addition of water (up to $33 \mathrm{wt} \%$ ) increased the concentration of heavy water in the montmorillonite interlayer and thus reduced further the peak height by reducing the contrast. However, a diffraction peak at a different position was observed, corresponding to an interlayer distance of $18.9 \pm 0.1 \AA$ in the raw plug material and $19.5 \pm 0.2 \AA$ in the polymerised material. This suggests yet again a significant modification to the water arrangement mediated by the polymer. Devineu et al. [20] studied bentonite pellets under compaction and reported that their water saturated bentonite pellets reached montmorillonite layer spacing of 15.7 $\AA$, corresponding to a duo-layer of water and 18.2 $\AA$ for the triple-layer of water. Thus our data points to a similar arrangement of water molecules into dual and triple layer system, but with somewhat electrically modified montmorillonite surface layer, giving the different spacing.

The information provided by the peak shape suggests that the raw sample had a smaller variation in the interlayer spacing than its polymerised counterpart, as demonstrated by the peak-width. As expected, the introduction of the $\mathrm{CD}_{4}$ reduced the peak intensity of the hydrated raw sample by about $15 \%$, however strangely, no reduction was observed in the polymerised sample. Furthermore, the width of the polymerised samples are much broader, suggesting that although the interlayers are more randomly separated than in the raw sample, there are less open pores smaller than the separation of the interlayers, and they were already filled up by the water. But the real curiosity is seen in the ZAC measurement of the highest water exposed samples. When tested with $\mathrm{CD}_{4}$ at $18 \%$ water concentration, the peak intensity dropped by $32 \%$, suggesting a large number of open pores smaller than about $17 \AA$, yet addition of extra 15 wt\% of water into this polymerised sample reduced the intensity by $23 \%$ and adding the ZAC gas did not increase this any further. Taking into account that the this height reduction was due largely from the alteration of the $\mathrm{H}$ to $\mathrm{D}$ concentration in the interlayer water, the lack of additional drop in intensity suggests that there is no open pores smaller than the separation of the interlayers, that is $19.6 \AA$. Thus it appears that the polymer enhances the movement and resizing of the aggregates in the clay in the presence of excess water and fills these pores preventing access to the gas.

\subsection{Bentonite Aggregates}

As already shown in the results section, the combined SANS and USANS inten- 
sity plots approximated straight lines when plotted in the logarithmic intensity plot. With the exception of the high Q-region, where the inter montmorillonite spacing dominates. Thus the data obeys the power law indicating chaotic system of contrasting pores, channels and low $S L D$ structures formed by the clay material on a large range of spatial scales.

The oven dried samples were investigated first. Figure 11 shows the fitted data.

The data is linear over almost the entire $Q$-range probed. There was however a sudden change in the slope of the line of best fit corresponding to a size of about $145 \mathrm{~nm}$ and $130 \mathrm{~nm}$ for the raw and polymerised bentonite respectively. The slope of the line below the transition point is almost identical to both samples ( -3.37) suggesting a material composed of clusters of particles (aggregates) of a diameter up to about $130 \mathrm{~nm}$ with a relatively rough, porous surface.

These small aggregates then form a structure of somewhat limited contact area best described by a mass fractal. Yoonessi et al. attributed this fractality to the presence of platelets and tactoids of various thicknesses, which are bent and curved [17]. The addition of the polymer appears to enhance this mass fractality, most likely by enhancing porosity on this scale, as the fractal dimension index (slope) is significantly less for the polymerised sample. Moreover, the departure from linearity at small $Q$ ss (structures greater than $\sim 1.4 \mu \mathrm{m}$ ), suggests that the polymer separates these clusters of fine aggregates further at larger scales, reducing contact area between them.
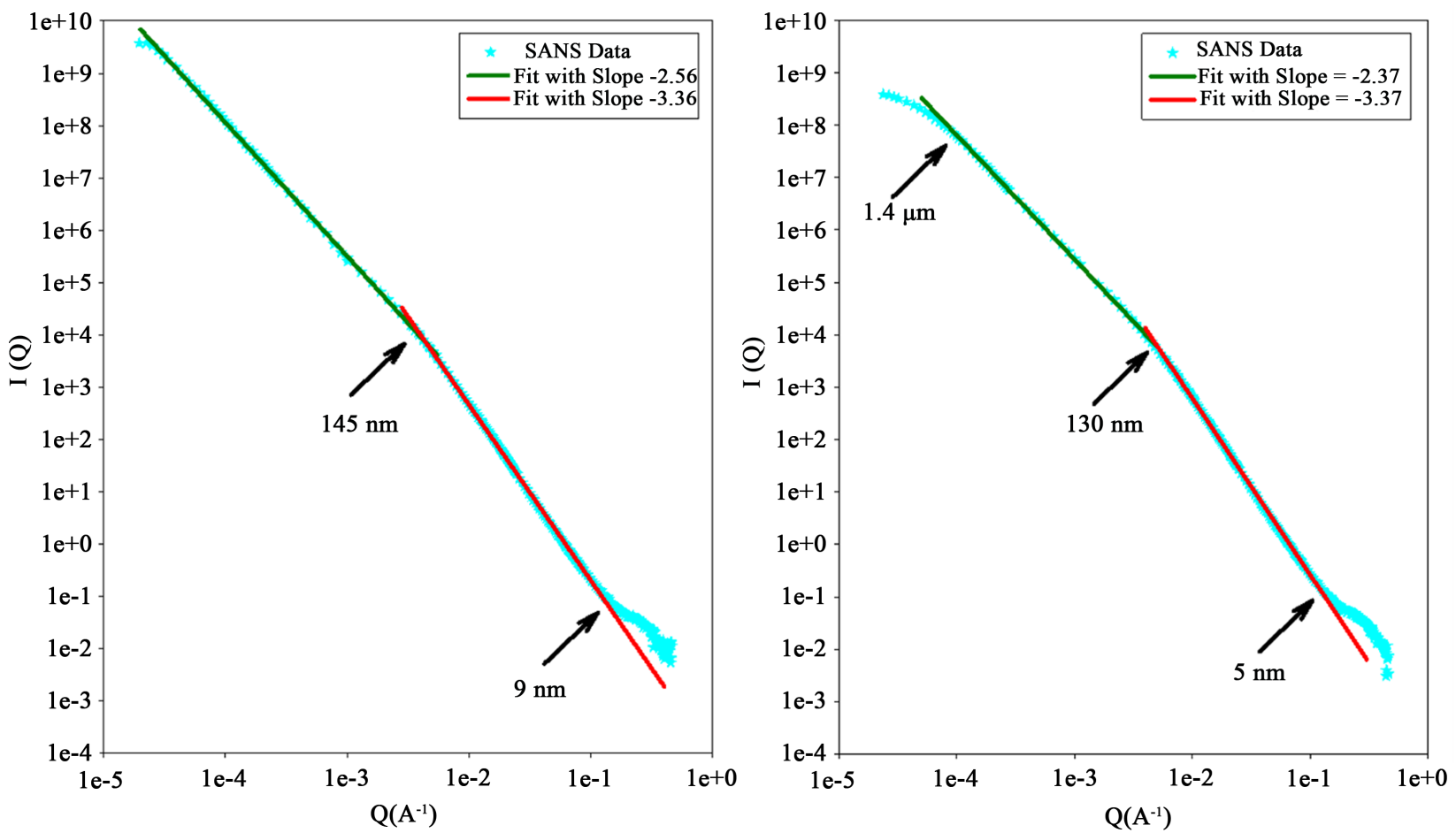

Figure 11. Intensity plots fitted with lines of best fit for the raw (left) and polymerised (right) bentonite plugs. The data was modelled with two straight lines on a log-log scale representing the power law. The high $Q$ data was fitted with a Gaussian previously (see Figure 17). The junction point of the two straight lines may represents the sizes of the tactoids making up the structure of the material. 
The shape of the SANS data shown in Figure 11 is unusual, as prior studies of bentonite clays did not report the dramatic change in slope seen around $140 \mathrm{~nm}$ [17] [21]. In fact the prior studies reported continuous mass fractality spanning the full range probed, thus we believe the formation of the particles smaller than $140 \mathrm{~nm}$ responsible for the surface fractality is due to the compaction process during the manufacturing of bentonite.

As mentioned in the Kratky analysis, the application of the deuterated methane at Zero Average Contrast (ZAC) pressure to these samples should in principle fill up the all gas accessible space in the them and thus reduce the scattering to zero unless some parts of the sample can't be accessed and some contrast is maintained. Figure 12 shows that on average intensity drop of close to $90 \%$ were measured, meaning that $10 \%$ of the sample volume was inaccessible.

Despite both samples being made of essentially the same bentonite, the addition of the polymer greatly changed the underlying structure of these inaccessible regions. The region of the intensity plot corresponding to the smallest scatterers up to $20 \mathrm{~nm}$ in diameter, consisted of isolated scatterers (pores) in the raw bentonite (fractality index of 2), yet the presence of the binder linked a substantial number of these into an interconnected network (increasing the fractality index to 2.4). This suggests that in the dry state, the binder is responsible for sealing off sections of the tactoids, preventing the gas from condensing in these small pores.
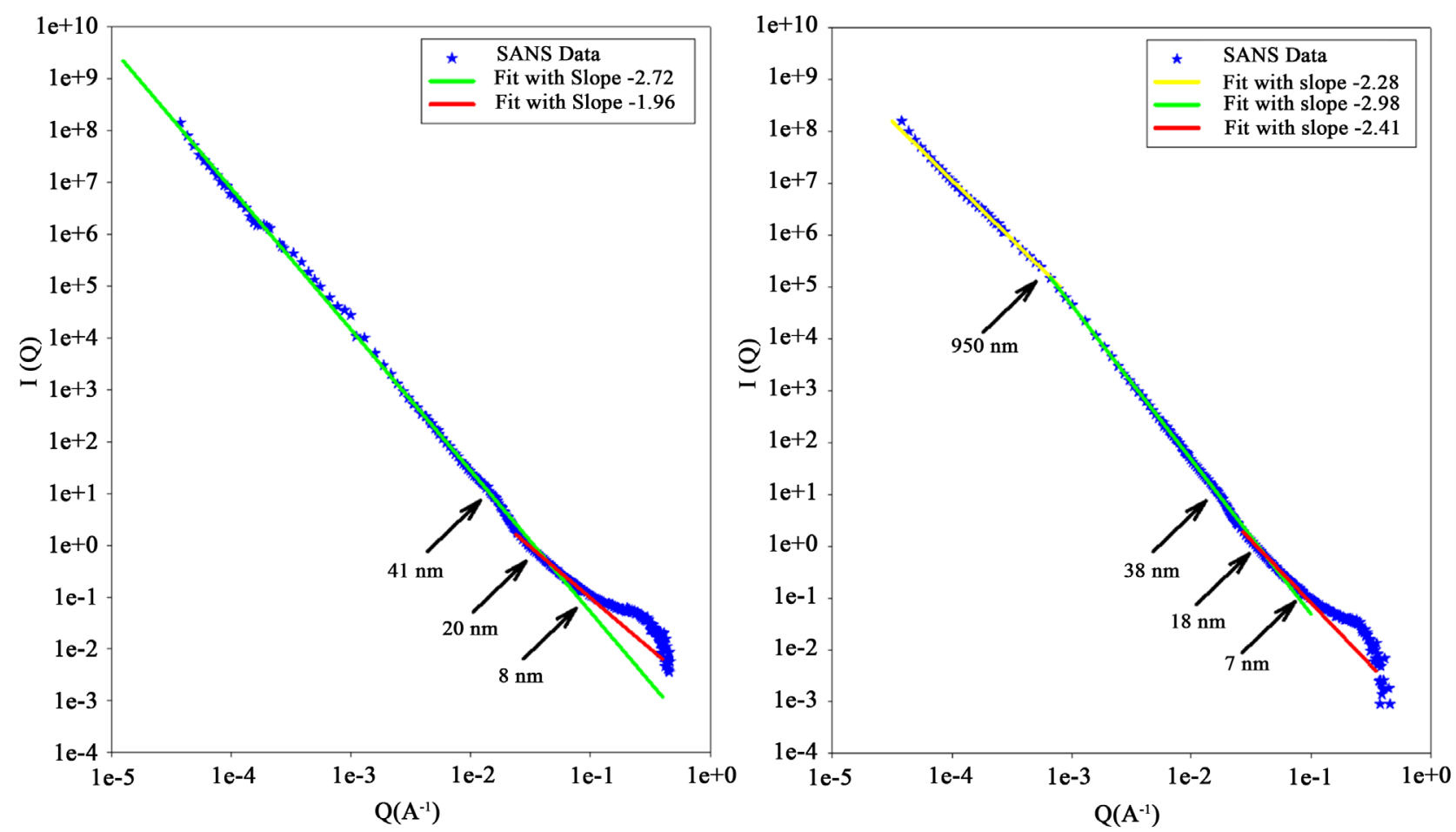

Figure 12. Intensity plots fitted with lines of best fit for raw (left) and polymerised (right) bentonite plugs saturated with deuterated methane at ZAC pressure. This data originates from the inaccessible pores and regions of the sample unaffected by the presence of the gas. This signal is only about 10 the intensity of the ambient sample. The underlying structure shows that the presence of the polymer profoundly affects this matrix. 
At scales larger than about $20 \mathrm{~nm}$, the curve changes slope and while the inaccessible structures in the raw bentonite remained a weakly interacting mass fractal, the polymer binder acts as a flexible medium that partially increases the surface contact between these inaccessible pores, giving the structure an overall surface fractality index 3 . This holds true up to about $1 \mu \mathrm{m}$, where on larger scales the structure become a network of weakly interacting particles best described by a mass fractal. This is not the nature of the surface of the clay, as the interface between the clay and the surrounding gas is contrasted out, but rather the data represents the pores and channels blocked off by either impurities or clay aggregates that prevent the high pressure $\mathrm{CD}_{4}$ from filling them. This provides information on the inaccessible porosity of the clay [14] [16].

At around $40 \mathrm{~nm}$ there is a hint of a bump in the intensity curve, suggesting some finer structure exists in this inaccessible network of pores. This structure is visible in both types of plug material. It may suggest that it is the result of some underlying inaccessible pore structure formed by trapped bubbles of air or other impurities with a sufficiently different $S L D$ to the clay average, producing the observed scattering. In the ambient condition, this structure is not visible due to overwhelming scattering from the bentonite itself and thus its contribution is "drowned out".

This assumption is further supported by the fact that it is visible in all the samples that were treated by the addition of a "contrast matching" fluid or gas, as seen in Figure 13 and Figure 15. This implies that whatever formed this structure, isobaric compression of $48.3 \mathrm{MPa} / 7000$ psi does not shift their position in $Q$-space, suggesting that this in fact may be due to some other hard spherical impurity that has sufficiently different $S L D$ as to produce a scattering there.

Hydration of the samples with contrast matched water showed that the material underwent significant changes with the uptake of water. Figure 13 shows the effect on both polymerised and raw samples when $18 \mathrm{wt} \%$ and $33 \mathrm{wt} \%$ of water was added to the material. The point of inflexion around $140 \mathrm{~nm}$ disappeared and was replaced by a straight line stretching from scatterers with diameter less than $10 \mathrm{~nm}$ to about $3 \mu \mathrm{m}$. These features are summarised in Figure 14.

In the high $Q$-range, that is the scatterers with a diameter of less than $10 \mathrm{~nm}$, in both the raw and polymerised hydrated samples, the materials are mass fractals. When the Bragg peak shown in the previous section is removed, this portion of the intensity plot fits a mass fractal, suggesting that the contribution here comes mostly from the inaccessible regions, embedded in the clay matrix. As we expect the open pores to be initially water-free, any water introduced to them would be with the ZAC ratio of $\mathrm{H}$ to $\mathrm{D}$ and so effectively removing any scattering from them. Further addition of $\mathrm{CD}_{4}$ at $\mathrm{ZAC}$ pressure shows that the absolute intensity over this region is almost the same, supporting the claim that the contribution here is mostly from the scatterers inaccessible to both water and high pressure methane. 


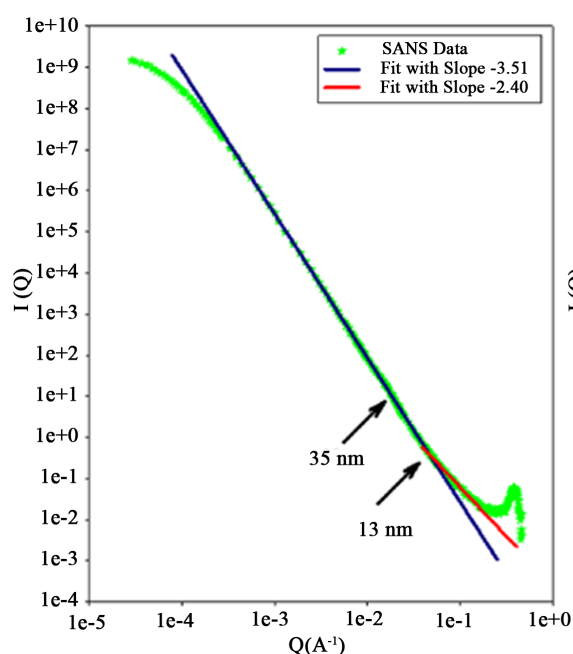

(a)

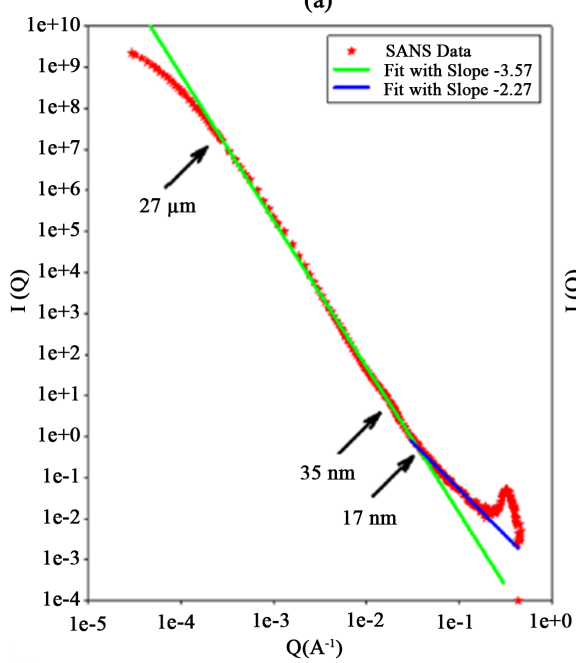

(c)

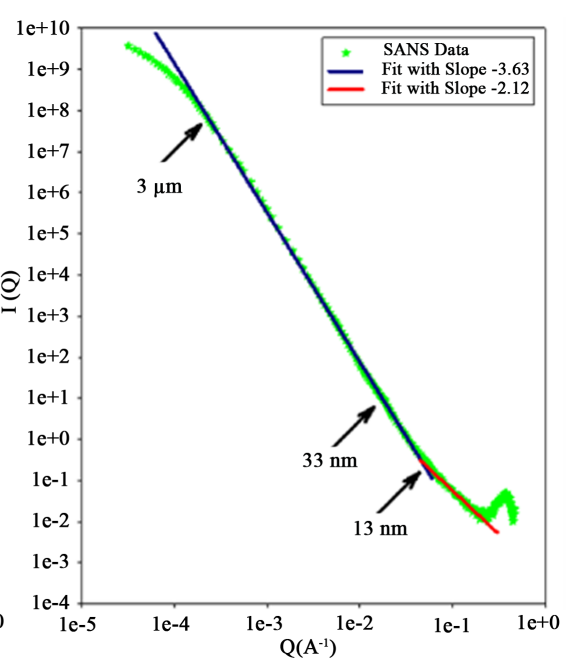

(b)

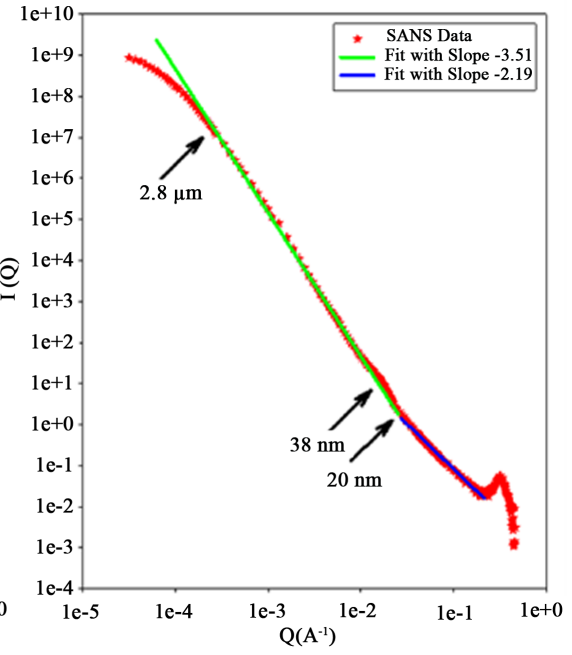

(d)

Figure 13. Intensity plots fitted with lines of best fit for hydrated samples. The data for raw (a) and polymerised (b) samples hydrated to $18 \mathrm{wt} \%$ are shown first, followed by again raw (c) and polymerised (d) samples hydrated to $33 \mathrm{wt} \%$ are plotted. As expected the biggest impact of the water is on the high $Q$-region of the intensity plot, where the addition of water fills the pores that $\mathrm{CD}_{4}$ filled.

The wetted samples then behaves as a surface fractal, suggesting that the contact area between the aggregates, so distinctly seen in the dry sample is now gone and the sample is a more compacted structure with a rough, open surface. Referring back to Figure 5, provides additional clues. When all the curves are directly compared, it is clear that pressurisation with $\mathrm{CD}_{4}$ has practically no effect on the intensity plot at sizes below the hump of $\sim 40 \mathrm{~nm}$, however the curves diverge in the smaller $Q$ 's. This shows that in these clays, the water in concentrations up to $33 \mathrm{wt} \%$ pools and fills completely the open pores smaller than $40 \mathrm{~nm}$. The pooled water does not affect pores greater than about $600 \mathrm{~nm}$ directly, as the ratio of ambient and ZAC plots are not affected in the raw material. But in the polymerised samples, in fact the intensity increases proportionally with the water intake. This suggests that larger open "channels" may be forming during the hy- 
dration process itself at the expense of the smaller ones, which are filled up with water and the removed tacktoids. These new, fine structures thus form tightly packed particles that grow in size and no longer are open to the gas intrusion. The surface fractality index increases until eventually it approaches the value of 4 indicating a hard, smooth interface between the clay and the surrounding medium may eventually form.

This process shows the nature of the self-healing reported for bentonite and indicates that the addition of the polymer enhances this process. The creation of the bigger channels needed to move water more effectively to the smaller pores (channels) is most likely accomplished by the polymer itself undergoing reconfiguration in the presence of water. A study that investigates this process in a greater detail would test this hypothesis and may be a focus of a future study.

Pressurisation of the wetted samples with $\mathrm{ZAC} \mathrm{CD}_{4}$ shows that the system of inaccessible pores/scatterer is hardly affected by the intake of water. The network of these pores forms mostly a mass fractal. This is summarised in Figure 15 and shows that the polymerised sample had to be fitted with three lines, but
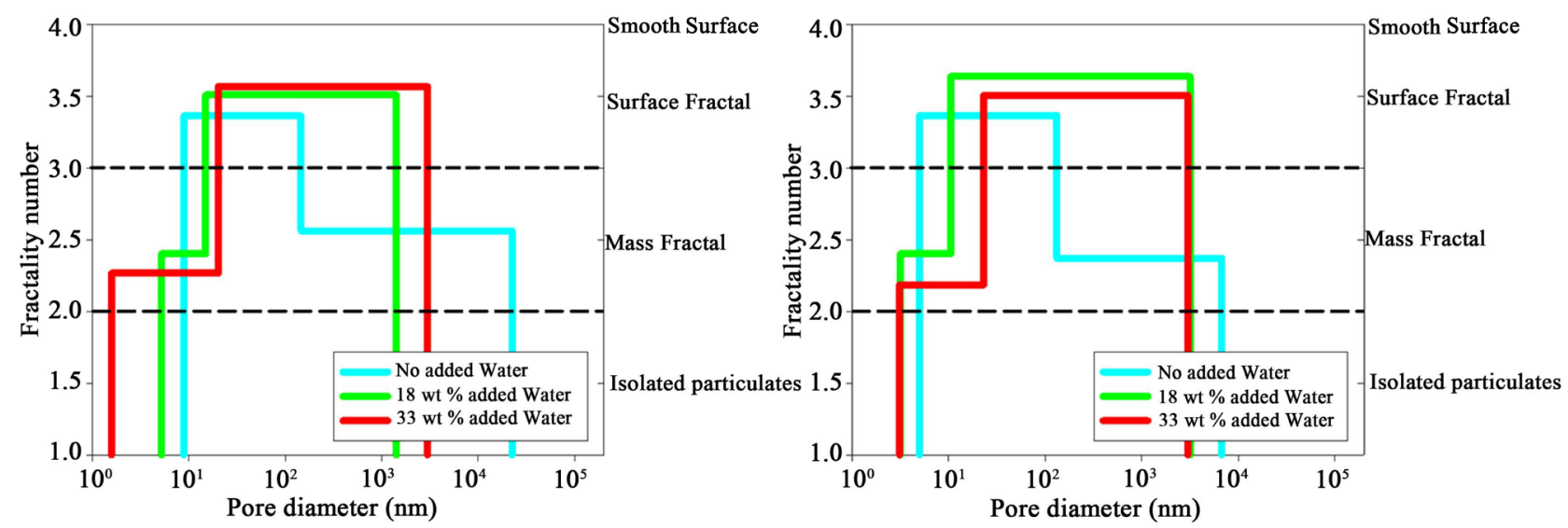

Figure 14. Fractality index and the spatial dimension it spans for the six samples measured. Raw bentonite data (left), polymerised samples (right).
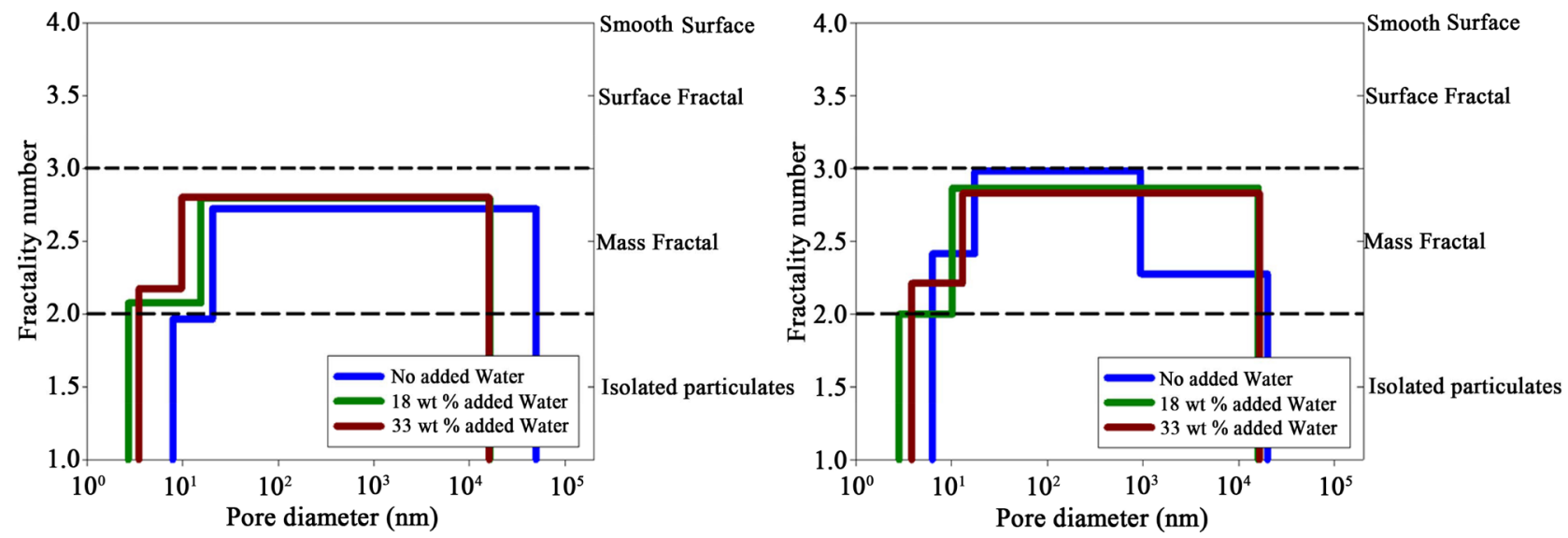

Figure 15. Fractality index and the spatial dimension they span of the closed pore network in raw (left) and polymerised (right) samples. Addition of water appears to clump the larger inaccessible pores together. 
that was no longer needed when the water was introduced. The inaccessible pores are isolated at the smallest scales, but form some interaction in the nano-range, and become more isolated again in the micro-range, suggesting that these are composed of larger, isolated regions with very rough surfaces. But these structures are affected by the rearranging clay structure and it appears that they may "clump" and undergo size change.

The creation of a second "hump" in the low Q-region, which centers around 3 $\mu \mathrm{m}$ with the initial uptake of water and then moves to higher $Q$ 's as water is added, creates smaller structures, perhaps helped by the process of healing. Figure 16(d) shows that the hump become very broad and its center moved from 3 $\mathrm{mm}$ down to $600 \mathrm{~nm}$ indicating that these inaccessible pores are being compacted and most likely clumped closer together with the addition of water and polymer.

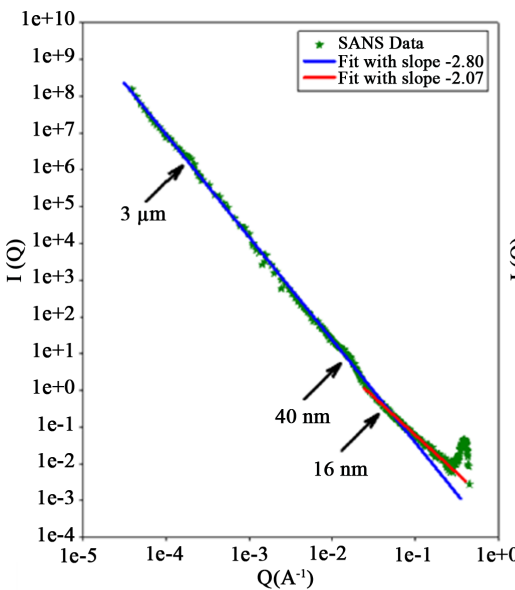

(a)

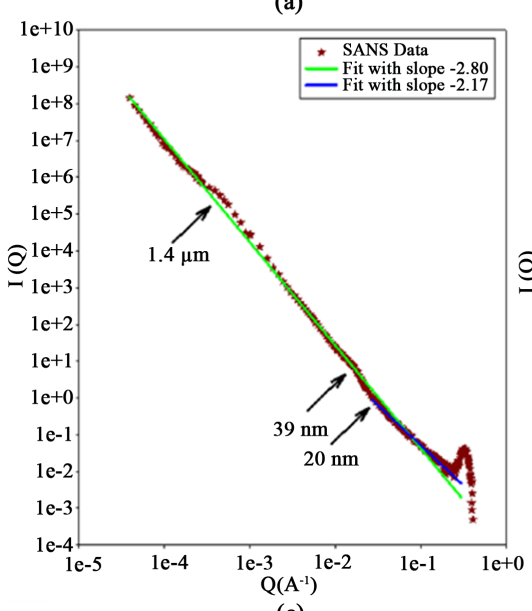

(c)

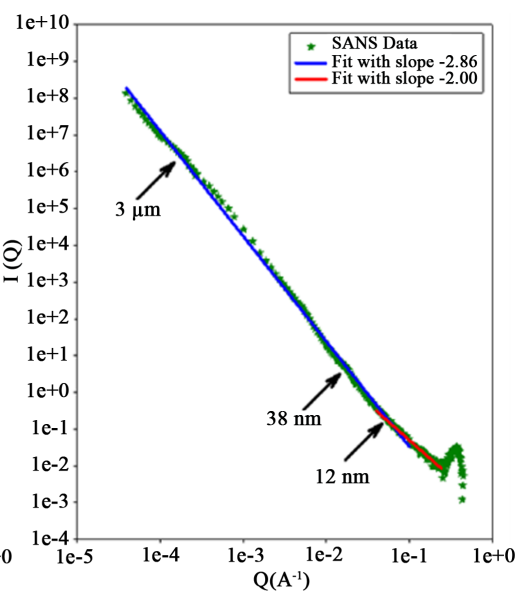

(b)

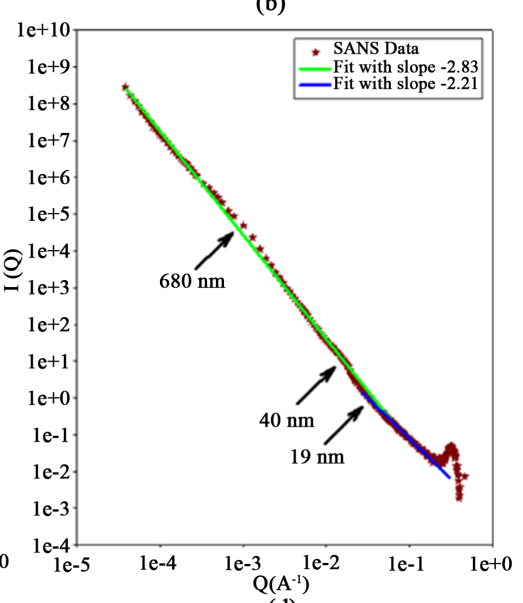

(d)

Figure 16. Intensity plots fitted with lines of best fit for raw ((a): 18 wt\% moisture content) and polymerised ((b): $18 \mathrm{wt} \%$ moisture content) bentonite plugs saturated with deuterated methane at ZAC pressure ((c): raw bentonite, 33 wt\% moisture content; d: polymerised bentonite, $33 \mathrm{wt} \%$ moisture content). This data originates from the inaccessible pores and regions of the sample unaffected by the presence of the gas. This signal is only about 10 the intensity of the ambient sample. The underlying structure shows that the presence of the polymer profoundly affects this matrix. 


\subsection{The Micron-Sized Structures}

The USANS study of the extra samples was reported in Figure 6 and Figure 7. Inspection of this data showed that there were significant differences in scattering intensities in the polymerised and raw samples as a function of water intake, particularly in the latter sample. However, when $\mathrm{CD}_{4}$ was added at the ZAC pressure, the data showed that most of the pores and channels are open structures and only minute changes already discussed in the previous section were noted.

The curves of Figure 6 and Figure 7 were fitted to the power law over the linear component of the curve and the summarized results are shown in Figure 17. At the sub-micron scale, in ambient conditions, both samples are mass fractals representing a weakly interacting collection of aggregates. With the initial uptake of water (9 $\mathrm{wt} \%$ ) the sample's larger aggregates segregate further and the sample become a stronger mass fractal as a consequence. However, this is not the case in the polymerised sample, where there appears to be very little change in the fractality index. The application of a 6 tone uniaxial strain on the sample has a surprising effect on the raw sample, as the mass fractality increased slightly, but had the opposite effect on the polymerised sample. This suggests that the addition of $9 \mathrm{wt} \%$ of water did not provide sufficient lubrication for the bulk of the sample to compact sufficiently to alter its structure on the sub-micron level and increase the contact between aggregates in this range. The opening of the bigger channels in the polymerised sample seen in the previous section does make the structure more sensitive to water it seems, as this sample underwent greater compaction as evidenced by the reduction in mass fractility.

Due to limited availability of the SANS machine, we could not complete these measurements and get the data for the nanoscale effects. Consequently the question of how mechanical compaction affects the nano-pores remains unanswered at this time.

Inspection of the non-linear component of the data in Figure 6 and Figure 7 also show that although the effect of mechanical compaction on the weakly hydrated
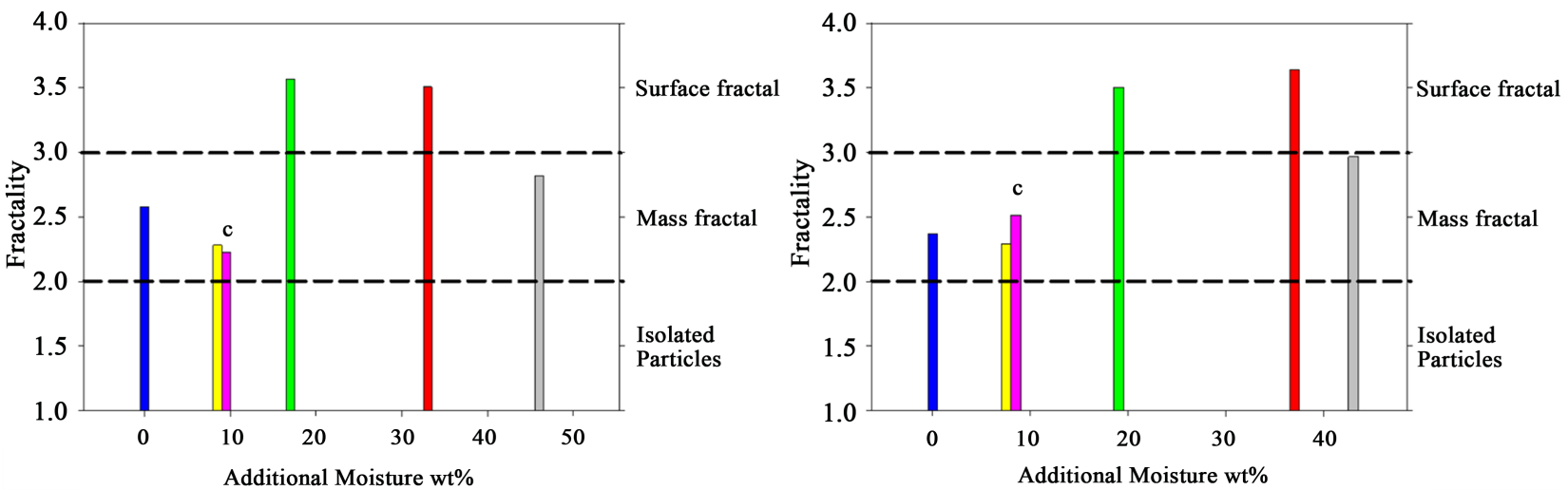

Figure 17. Fractality index measured for the raw (left) and polymerised samples (right) as measured on USANS. In the sub-micron size resolution. The effect of uniaxial compression is shown in the $\mathrm{C}$ bar. The grey bar with maximum moisture concentration was obtained using an eye-dropper to introduce the water directly to the sample. 
samples was small for scatterers below a micron, at sizes above a micron, the polymerised and non-polymerised samples behaved very differently. The addition of the polymer bonded the sample under mechanical compression more tightly, as indicated in the flattening of the intensity plot in the smallest $Q$-range. The upper limit on scatterer size was lost, purely due to compression during manufacture, but definitely due to different plug composition, as the raw, dry sample did not experience any flattening.

The application of the uniaxial strain on the $9 \mathrm{wt} \%$ sample showed interesting behavior. Scattering from the pores greater than 1 micron remained almost unchanged in the raw sample, but has dramatically increased in the polymerised sample. This suggests that compression deformed the existing big pores, resizing them and hence creating larger pores with no capping off limit, as the compressed curve does not depart from the power law fit shown in Figure 6 (left), Figure 7 (left) and Figure 17. The increase thus must be due to polymer induced deformation and the creation of extra-large pores, as the $\mathrm{CD}_{4} \mathrm{ZAC}$ measurement did not detect any changes in the inaccessible pore structures.

The liquid vs vapour hydrated sample showed that the fractal index fell to below the surface fractal index, suggesting that the material started to separate. During vapour hydrogenation the water was entering the sample slowly, over the $48 \mathrm{hr}$ period, but this sample was exposed to an interaction of liquid water from an eye-dropper and thus the clay was able to form the aggregates faster and delaminate where the water was pooled. It most likely accelerated the "healing" process and hence the mass fractal nature of the sample.

\section{Conclusions}

This article shows the application of the SANS/USANS technique to problems involving nanostructures. In combination with the customised cell, it is possible to observe the changes in microstructure as a function of moisture content, strain and other chemical and physical perturbation. The technique offers a cheaper alternative to full in-field testing and is well suited for small model, long term testing. This study shows:

- Evolution of structural changes in the plug material as a function of moisture and polymer content

- Evolution of porosity changes and the effect of moisture on the plug material

- Nano-structures in the plug material most affected by the moisture and polymer addition

- Mechanism of self-healing

The addition of the polymer binder into the bentonite plugging material alters the physical properties of the plug material. On the finest scale, the dry materials exhibited fine and relatively flexible laminar structure separated by about $22.7 \AA$. With the introduction of an isotopically compressing gas into the polymerised and un-polymerised samples, these layers swell by $11 \%$ in the first sample and compacted by $6 \%$ in the latter sample. No such effect was measured when mois- 
ture was introduced to the samples. In fact an addition of $18 \mathrm{wt} \%$ and further 15 $w t \%$ of water maintained a rigid 2 and 3 water molecule layer respectively in the interlayer space that was unaffected by the introduction of methane in all but the $18 \mathrm{wt} \%$ sample which swelled by $4 \%$ in the presence of methane.

Although the pressure of gaseous methane and the $S L D$ of $\mathrm{H}_{2} \mathrm{O} / \mathrm{D}_{2} \mathrm{O}$ mixture was carefully selected as to eliminate scattering from the plug material and introduced fluid interface, the intensity plot showed that about $90 \%$ of scattering originated from gas accessible pores and channels and these pores underwent slow reorganisation with the uptake of water.

On the submicron size, the polymer opened more pores and channels open to the influx of methane and the data suggested that the presence of the polymer helps in the self-healing process by stripping the finer aggregates and using them to closely fill up the small pores.

More beam time is required to investigate the effects of uniaxial strain and hydrogenation by liquid vs vapor, as only USANS was completed due to time limitation on the instrument.

These results will help to refine the hydration model developed at the University of Queensland and allows better prediction of the swelling behavior of bentonite plugs under wellbore conditions.

\section{Acknowledgements}

This research was funded by The University of Queensland Centre for Coal Seam Gas (UQ-CCSG) and its industry members (Arrow Energy, Australia Pacific LNG, Santos and Shell/QGC) and the Queensland Government Advance Queensland Innovation Partnerships program. Neutron beam time was provided by the Centre for Neutron Research, National Institute of Standards and Technology, Gaithersburg MD, USA. The high pressure gas equipment was provided by the GP-SANS beamline, HiFiR Reactor Group, Oak Ridge National Laboratory, Oak Ridge TN, USA. The authors would like to thank Dr Susana Teixeira and Dr David Mildner of NIST for their help in performing these measurements.

\section{Conflicts of Interest}

The authors declare no conflicts of interest regarding the publication of this paper.

\section{References}

[1] Working Group III of the Intergovernmental Panel on Climate Change (2003) IPCC Special Report on Carbon Dioxide Capture and Storage. Cambridge University Press, Cambridge.

[2] Department of Conservation (2006) Title 14, California Code of Regulations. California Department of Conservation.

[3] Barclay, I., Pellenbarg, J., Tettero, F., Pfeiffer, J., Slater, H., Staal, T., Stiles, D., Tilling, G. and Whitney, C. (2007) The Beginning of the End: A Review of Abandon- 
ment and Decommissioning Practices. Oilfield Review, 13, 28-41.

[4] Englehardt, J., Wilson, M.J. and Woody, F. (2001) New Abandonment Technology New Materials and Placement Techniques. SPE/ EPA/DOE Exploration and Production Environmental Conference, San Antonio, TX, 26-28 February 2001, SPE-66496. https://doi.org/10.2118/66496-MS

[5] Towler, B.F., Victorov, H., Zamfir, G. and Ignat, P. (2008) Plugging Wells with Hydrated Bentonite, Part 2: Bentonite Bars. SPE Annual Technical Conference and Exhibition, Denver, CO, 21-24 September 2008, SPE-115524. https://doi.org/10.2118/115524-MS

[6] Towler, B.F., Firouzi, M., Holl, H.-G., Gandhi, R. and Thomas, A. (2016) Field Trials of Plugging Oil and Gas Wells with Hydrated Bentonite. SPE Asia Pacific Oil \& Gas Conference and Exhibition, Perth, Australia, 25-27 October 2016, SPE-182199-MS. https://doi.org/10.2118/182199-MS

[7] Holl, H.-G. (2019) Hydraulic Testing of Compacted Bentonite Used for Plug and Abandonment Operations. Journal of Minerals and Materials Characterization and Engineering, 7.

[8] Holl, H.-G. and Scheuermann, A. (2018) Characterisation of Geomechanical Properties of Bentonite Clay Used for Plug and Abandonment Operations of Coal Seam Gas Wells. Journal of Minerals and Materials Characterization and Engineering, 6, 218-234. https://doi.org/10.4236/jmmce.2018.62016

[9] NIST (2018) Neutron SLD Calculator. NIST Centre for Neutron Research. https://www.ncnr.nist.gov/resources/activation/

[10] Karnland, O. (2010) Chemical and Mineralogical Characterization of the Bentonite Buffer for the Acceptance Control Procedure in a KBS-3 Repository. Technical Report.

[11] CETCO (2013) Sodium Bentonite: Its Structure and Properties. CETCO Pty Ltd., Canning Vale.

[12] Grim, R.E. (1968) Clay Mineralogy. McGraw Hill Book Company, New York.

[13] Masin, D. and Khalili, N. (2016) Swelling Phenomena and Effective Stress in Compacted Expansive Clays. Canadian Geotechnical Journal, 53, 134-147. https://doi.org/10.1139/cgj-2014-0479

[14] Melnichenko, Y.B. (2016) Small-Angle Scattering from Confined and Interfacial Fluids. Springer International Publishing, New York. https://doi.org/10.1007/978-3-319-01104-2

[15] Radlinski, A.P. and Boreham, C.J. (1996) Microstructural Evolution of Source Rocks during Hydrocarbon Generation: A Small-Angle-Scattering Study. Physical Review B, 53, 14152-14160. https://doi.org/10.1103/PhysRevB.53.14152

[16] Radlinski, A.P., Boreham, C.J., Lindner, P., Randl, O., Wignall, G.D., Hinde, A. and Hope, J.M. (2000) Small Angle Neutron Scattering Signature of Oil Generation in Artificially and Naturally Matured Hydrocarbon Source Rocks. Organic Geochemistry, 31, 1-14. https://doi.org/10.1016/S0146-6380(99)00128-X

[17] Yoonessi, M., Toghiani, H., Daulton, T., Lin, J. and Pittman Jr., C. (2005) Clay Delamination in Clay/Poly(Dicyclopentadiene) Nanocomposites Quantified by Small Angle Neutron Scattering and High-Resolution Transmission Electron Microscopy. Macromolecules, 38, 818-830. https://doi.org/10.1021/ma048663e

[18] Pusch, R. (2015) Bentonite Clay-Environmental Properties and Applications. CRC Press, Taylor \& Francis Group, Boca Raton, FL. https://doi.org/10.1201/b18543

[19] Segad, M., Jönsson, B., Åkesson, T. and Cabane, B. (2010) Ca/Na Montmorillonite: 
Structure, Forces and Swelling Properties. Langmuir, 26, 5782-5790. https://doi.org/10.1021/la9036293

[20] Devineu, K., Bihannic, I., Michot, L., Villieras, F., Masrouri, F., Cuisinier, O., Fragneto, G. and Michau, N. (2006) In Situ Neutron Diffraction Analysis of the Influence of Geometric Confinement on Crystalline Swelling of Montmorillonite. Applied Clay Science, 31, 76-84. https://doi.org/10.1016/j.clay.2005.08.006

[21] Bihannic, I., Delville, A., Deme, B., Plazanet, M., Villieras, F. and Michot, L.J. (2009) Clay Swelling: New Insights from Neutron-Based Techniques. In: Liang, L., Rinaldi R. and Schober, H., Eds., Neutron Applications in Earth, Energy and Environmental Sciences, Springer, New York, 521-546.

https://doi.org/10.1007/978-0-387-09416-8_18 